\title{
Existence Results for the Quasistationary Motion of a Free Capillary Liquid Drop
}

\author{
M. Günther and G. Prokert
}

\begin{abstract}
We consider instationary creeping flow of a viscous liquid drop with free boundary driven by surface tension. This yields a nonlocal surface motion law involving the solution of the Stokes equations with Neumann boundary conditions given by the curvature of the boundary. The surface motion law is locally reformulated as a fully nonlinear parabolic (pseudodifferential) equation on a smooth manifold. Using analytic expansions, invariance properties, and a priori estimates we give, under suitable presumptions, a short-time existence and uniqueness proof for the solution of this equation in Sobolev spaces of sufficiently high order. Moreover, it is shown that if the initial shape of the drop is near the ball, then the evolution problem has a solution for all positive times which exponentially decays to the ball.
\end{abstract}

Keywords: Stokes flows, quasisteady motions, surface tensions, nonlinear parabolic equations, surface motion laws

AMS subject classification: Primary $35 \mathrm{R} 35$, secondary $35 \mathrm{Q} 35,76 \mathrm{D} 07$

\section{Introduction}

In fluid dynamics problems with very small Reynolds number the concept of "creeping flow" is used. This means that the inertial forces are neglected and, in the case of Newtonian flow, the Navier-Stokes equations simplify to the Stokes equations. When one uses such a simplification to describe liquid motions that are actually instationary, it could be called a quasistationary approximation. This idea is the basis for the following model of the motion of a viscous liquid drop under the influence of capillary forces which is successfully used in the description of the so-called viscous sintering process in glass production [21].

The liquid is assumed to be incompressible and to have constant viscosity, density, and (positive) surface tension coefficient. The only driving mechanism we consider is the force from surface tension. In dimensionless form this leads to the linear boundary

M. Günther: Leipzig University, Institute of Mathematics, Augustusplatz 10-11, 04109 Leipzig, Germany. Research partially supported by the Deutsche Forschungsgemeinschaft. e-mail: guenther@mathematik.uni-leipzig.d400.de

G. Prokert: Technical University Eindhoven, Faculty of Mathematics and Computer Science, P.O. Box 513, 5600 MB Eindhoven, The Netherlands; e-mail: prokert@win.tue.nl 
value problem

$$
\left.\begin{array}{rl}
-\Delta u+\nabla p & =0 \\
\operatorname{div} u & =0
\end{array}\right\} \quad \text { in } \Omega(t)
$$

where $\Omega(t) \subset \mathbb{R}^{N}$ is the (bounded) domain occupied by the drop at time $t \geq 0, u$ and $p$ are the velocity and pressure fields in $\Omega(t)$ at this time,

$$
\mathcal{T}(u, p)=\left((\nabla u)+(\nabla u)^{T}\right)-p I
$$

denotes the stress tensor, $\kappa_{t}$ and $n_{t}$ denote the double mean curvature and the outer normal vector of $\Gamma(t)$. The sign of $\kappa_{t}$ is taken such that it is negative if $\Omega(t)$ is convex.

As will be shown below, the equations (1.1) essentially determine $u$ and $p$ at time $t$. For the description of the motion of the drop the kinematic boundary condition

$$
V_{n}(t)=\left.u\right|_{\Gamma(t)} \cdot n_{t} \quad \text { on } \Gamma(t)
$$

has to be added where $V_{n}(t)$ denotes the normal velocity of $\Gamma(t)$. This condition is an equivalent expression for the demand that the set of particles that constitute the boundary of the drop does not change in time.

The problem (1.1), (1.2) is a moving boundary problem that can be considered as a problem of evolution of $\Gamma=\Gamma(t)$ by a nonlocal surface motion law, comparable, e.g., to Hele-Shaw flow driven by surface tension [7 - 9]. The problem (1.1), (1.2) and its counterpart concerning outer domains, which is a model for bubbles in a viscous liquid, have recently been investigated in the two-dimensional case. This has been done by methods from complex function theory, using, in particular, time-dependent conformal mappings and the solution of Hilbert problems $[3,4,14,15,18]$. For the numerical treatment of the problem we refer to [21] and the bibliography therein.

The aim of this paper is to provide an analysis of this problem in $N$ dimensions (for the sake of simplicity sometimes restricted to $N=3$ ) as far as this can be done by local methods. Accordingly, we prove, under suitable presumptions, a short-time existence and uniqueness result for general initial domains and global existence and exponential decay of the solution near. the stable equilibrium solutions that are given by the balls.

Notation. All differentiations with exception of those with respect to the time variable $t$ are to be understood in generalized sense. We will use the symbols $C$ and $c$ for "large" and "small" positive real constants, respectively. Sometimes an index is used to indicate their dependence on parameters. A function that is given on a (sufficiently regular) domain $\Omega$ and its restriction or trace at the boundary $\Gamma$ of this domain are often denoted by the same symbol. The norms in the Sobolev spaces $H^{s}(\Omega)$ and $H^{s}(\Gamma)$ are denoted by $\|\cdot\|_{s}^{\Omega}$ and $\|\cdot\|_{s}^{\Gamma}$, respectively, and the same notation is used for the norms of the corresponding Sobolev spaces of vector-valued functions $\left(H^{s}(\Omega)\right)^{k}$ and $\left(H^{s}(\Gamma)\right)^{k}$. (These norms are specified later, at the moment it is sufficient to demand that they generate the usual topologies.) 
For convenience we generalize some notions of vector algebra and analysis to $\mathbb{R}^{N}$. Let $K$ be an arbitrary but fixed bijection from the set $\{(i, j) \mid I \leq i<j \leq N\}$ to the set $\left\{1, \ldots,\left(\begin{array}{c}N \\ 2\end{array}\right)\right\}$. We define the bilinear mappings

$$
\begin{aligned}
& \times: \mathbb{R}^{N} \times \mathbb{R}^{N} \longrightarrow \mathbb{R}^{\left(\begin{array}{c}
N \\
2
\end{array}\right)} \\
& \otimes: \mathbb{R}^{\left(\begin{array}{l}
N \\
2
\end{array}\right)} \times \mathbb{R}^{N} \longrightarrow \mathbb{R}^{N}
\end{aligned}
$$

by

$$
(a \times b)_{K(i, j)}=a_{i} b_{j}-a_{j} b_{i} \quad(1 \leq i<j \leq N)
$$

and

$$
(c \otimes a)_{i}=\sum_{j=1}^{i-1} c_{K(j, i)} a_{j}-\sum_{j=i+1}^{N} c_{K(i, j)} a_{j} \quad \cdot(i=1, \ldots, N) .
$$

It is easy to check that

$$
c \cdot(a \times b)=b \cdot(c \otimes a) \quad \forall a, b \in \mathbb{R}^{N}, c \in \mathbb{R}^{\left(\begin{array}{c}
N \\
2
\end{array}\right) .}
$$

We define, moreover, for any sufficiently smooth $N$-vector function $v$ given on an open subset of $\mathbb{R}^{N}$, the $\left(\begin{array}{c}N \\ 2\end{array}\right)$-vector-valued differential operator rot by

$$
(\operatorname{rot} v)_{k(i, j)}=\frac{\partial v_{j}}{\partial x_{i}}-\frac{\partial v_{i}}{\partial x_{j}} \quad(1 \leq i<j \leq N)
$$

for which we have the integral theorem

$$
\int_{\Omega} \operatorname{rot} v d x=\int_{\Gamma} n \times v d \Gamma
$$

Note that if $N=3$, then the usual definitions of the outer product and the curl (rotation) of a vector field can be obtained, up to the sign of the second component, by choosing the suitable bijection $K$.

\section{The boundary value problem on a fixed domain}

We consider the boundary value problem

$$
\begin{aligned}
& \left.\begin{array}{rl}
-\Delta u+\nabla p & =0 \\
\operatorname{div} u & =0
\end{array}\right\} \text { in } \Omega \\
& \mathcal{T}(u, p) n=\kappa n \quad \text { on } \Gamma=\partial \Omega
\end{aligned}
$$

on a bounded smooth domain $\Omega \subset \mathbb{R}^{N}$ that is taken fixed in this section. The quantities $\kappa$ and $n$ are defined analogously to $\kappa_{t}$ and $n_{t}$ in (1.1). 
At first we establish the unique solvability of a generalized weak formulation of (2.1) with auxiliary conditions. We introduce the Hilbert spaces

$$
\begin{aligned}
& X=\left(H^{1}\left(\Omega_{0}\right)\right)^{N} \times L^{2}\left(\Omega_{0}\right) \times\left(\mathbb{R}^{N} \times \mathbb{R}^{\left(\begin{array}{c}
N \\
2
\end{array}\right)}\right) \\
& Y=\left(\left(H^{1}\left(\Omega_{0}\right)\right)^{N}\right)^{\prime} \times\left(L^{2}\left(\Omega_{0}\right) \times \mathbb{R}^{N} \times \mathbb{R}^{\left(\begin{array}{c}
N \\
2
\end{array}\right)}\right)
\end{aligned}
$$

and the (bi-)linear operators

$$
\begin{aligned}
L & : X \longrightarrow Y \\
A & :\left(H^{1}(\Omega)\right)^{N} \longrightarrow\left(\left(H^{1}(\Omega)\right)^{N}\right)^{\prime} \\
B & :\left(H^{1}(\Omega)\right)^{N} \longrightarrow L^{2}\left(\Omega_{0}\right) \times\left(\mathbb{R}^{N} \times \mathbb{R}^{\left(\begin{array}{c}
N \\
2
\end{array}\right)}\right) \\
a & :\left(H^{1}(\Omega)\right)^{N} \times\left(H^{1}(\Omega)\right)^{N} \longrightarrow \mathbb{R} \\
\varphi_{1} & :\left(H^{1}(\Omega)\right)^{N} \longrightarrow \mathbb{R}^{N} \\
\varphi_{2} & :\left(H^{1}(\Omega)\right)^{N} \longrightarrow \mathbb{R}^{\left(\begin{array}{c}
N \\
2
\end{array}\right)}
\end{aligned}
$$

defined by

$$
\begin{aligned}
L\left[\begin{array}{l}
u \\
p \\
\lambda
\end{array}\right] & =\left[\begin{array}{c}
A u+B^{\prime}\left[\begin{array}{l}
p \\
\lambda
\end{array}\right] \\
B u
\end{array}\right] \\
(A u) v & =a(u, v) \\
B u & =\left[\begin{array}{c}
-\operatorname{div} u \\
\varphi_{1}(u) \\
\varphi_{2}(u)
\end{array}\right] \\
a(u, v) & =\frac{1}{2} \int_{\Omega}\left(\frac{\partial u_{i}}{\partial x_{j}}+\frac{\partial u_{j}}{\partial x_{i}}\right)\left(\frac{\partial v_{i}}{\partial x_{j}}+\frac{\partial v_{j}}{\partial x_{i}}\right) d x \\
\varphi_{1}(u) & =\int_{\Omega} u d x \\
\varphi_{2}(u) & =\int_{\Omega} \operatorname{rot} u d x
\end{aligned}
$$

where $B^{\prime}: L^{2}(\Omega) \times\left(\mathbb{R}^{N} \times \mathbb{R}^{\left(\begin{array}{c}N \\ 2\end{array}\right)}\right) \longrightarrow\left(\left(H^{1}\left(\Omega_{0}\right)\right)^{N}\right)^{\prime}$ is the dual of $B$.

Lemma 1 (Weak formulation).

(i) The operator $L$ is a homeomorphism between $X$ and $Y$.

(ii) Suppose $L[u p \lambda]^{T}=[f 0]^{T}$ with

$$
f(v)=\int_{\Gamma} \kappa n \cdot v d \Gamma \quad \forall v \in\left(H^{1}(\Omega)\right)^{N} .
$$

Then $\lambda=0$ and $(u, p)$ is a weak solution of. (2.1). 
Proof. Statement (i): The equation

$$
L[u p \lambda]^{T}=F
$$

is a variational problem with linear restrictions to which the usual existence results apply (see, e.g., [6]). In order to establish (i) it is therefore sufficient to show that $a$ is elliptic on ( $\operatorname{ker} B,\|\cdot\|_{1}^{\Omega}$ and $B$ is surjective.

The first statement follows from Poincarés inequality [10]

$$
\int_{\Omega}|\nabla w|^{2} d x+\left(\int_{\Omega} w d x\right)^{2} \geq c\|w\|_{1}^{\Omega^{2}} \quad \forall w \in H^{1}(\Omega)
$$

and Korns second inequality [11]

$$
a(v, v) \geq c \sum_{i, j=1}^{N} \int_{\Omega}\left(\frac{\partial v_{i}}{\partial x_{j}}\right)^{2} d x \quad \forall v \in\left\{v \in\left(H^{1}(\Omega)\right)^{N} \mid \varphi_{2}(v)=0\right\}
$$

Taking into account that $\varphi_{1}$ and $\varphi_{2}$ are surjective from $\left\{v \in\left(H^{1}(\Omega)\right)^{N} \mid \operatorname{div} v=0\right\}$ to $\mathbb{R}^{N}$ and $\mathbb{R}^{\left(\begin{array}{c}N \\ 2\end{array}\right)}$, respectively, it remains to show that the equation $-\operatorname{div} v=q$ in $\Omega$ is solvable in $\left(H^{1}(\Omega)\right)^{N}$ for all $q \in L^{2}\left(\Omega_{0}\right)$. This can be done by considering a solution $\Phi \in H^{2}(\Omega)$ of $-\Delta \Phi=q$ in $\Omega$ and setting $v=\nabla \Phi$.

Statement (ii): Consider the space

$$
V_{0}=\left\{v \in\left(H^{1}(\Omega)\right)^{N} \mid v_{i}(x)=\sum_{j=1}^{N} s_{i j} x_{j}+c_{i}\left(s_{i j}, c_{i} \in \mathbb{R}, s_{i j}=-s_{j i}\right)\right\}
$$

$(i=1, \ldots, N)$ and note that $a(\cdot, v), a(u, \cdot)$, and div vanish on $V_{0}$. The same holds for $f$ because the Green formula for closed surfaces yields

$$
\int_{\Gamma} \kappa n \cdot v d \Gamma=\int_{\Gamma} \Delta_{\Gamma} x \cdot v d \Gamma=-\sum_{i=1}^{N} \int_{\Gamma} \nabla_{\Gamma} x_{i} \cdot \nabla_{\Gamma} v_{i} d \Gamma
$$

where $x_{i}$ and $v_{i}$ are the coordinates of $x$ and $v$ in a fixed Cartesian basis of $\mathbb{R}^{N}$, and $\nabla_{\Gamma}$ and $\Delta_{\Gamma}$ are the generalized gradient and the Laplace-Beltrami operator on $\Gamma$, respectively. Hence $\lambda=0$ because $\varphi_{1}$ and $\varphi_{2}$ are surjective from $V_{0}$ to $\mathbb{R}^{N}$ and $\mathbb{R}^{\left(\begin{array}{c}N \\ 2\end{array}\right)}$, respectively. The fact that in this case (2.2) is a weak formulation of (2.1) follows from the integral identity

$$
\begin{aligned}
\frac{1}{2} \sum_{i, j=1}^{N} & \int_{\Omega}\left(\frac{\partial u_{i}}{\partial x_{j}}+\frac{\partial u_{j}}{\partial x_{i}}\right)\left(\frac{\partial v_{i}}{\partial x_{j}}+\frac{\partial v_{j}}{\partial x_{i}}\right) d x-\int_{\Omega} p \operatorname{div} v d x \\
\quad & =\int_{\Omega}(-\Delta u+\nabla p) \cdot v d x-\int_{\Omega} \nabla(\operatorname{div} u) \cdot v d x+\int_{\Gamma} \mathcal{T}(u, p) n \cdot v d \Gamma
\end{aligned}
$$

holding for sufficiently smooth vector-valued functions $u, v$ and scalar functions $p$ 
Furthermore, we will need some $H^{s}$-regularity results on our boundary value problem. For fixed $s \geq 2$, we introduce the spaces

$$
\begin{aligned}
& \left.\tilde{X}=\left(H^{1}(\Omega)\right)^{N} \times\left(H^{s-1}(\Omega)\right)^{N} \times \mathbb{R}^{N} \times \mathbb{R}^{\left(\frac{N}{2}\right)}\right) \\
& \tilde{Y}=\left(H^{s-2}(\Omega)\right)^{N} \times H^{s-1}(\Omega) \times\left(H^{s-\frac{3}{2}}(\Gamma)\right)^{N} \times \mathbb{R}^{N} \times \mathbb{R}^{\left(\frac{N}{2}\right)}
\end{aligned}
$$

and the operator

$$
\tilde{L}: \tilde{X} \longrightarrow \tilde{Y}
$$

defined by

$$
\tilde{L}\left[\begin{array}{c}
u \\
p \\
\lambda
\end{array}\right]=\left[\begin{array}{c}
-\Delta u+\nabla p+\lambda_{1} \\
-\operatorname{div} u \\
\mathcal{T}(u, p) n+\lambda_{2} \otimes n \\
\varphi_{1}(u) \\
\varphi_{2}(u)
\end{array}\right]
$$

Lemma 2 (Regularity).

(i) The operator $\widetilde{L}$ is a homeomorphism between the spaces $\widetilde{X}$ and $\tilde{Y}$.

(ii) Suppose $\widetilde{L}[u p \lambda]^{T}=\left[00 F_{B} 00\right]^{T}$. Then

$$
\|\lambda\|_{\left.\mathbb{R}^{N} \times \mathbb{R}^{(} \frac{N}{2}\right)} \leq C_{\sigma}\left\|F_{B}\right\|_{\sigma}^{\Gamma}
$$

for all $\sigma \in \mathbb{R}$ and

$$
\|u\|_{0}^{\Omega} \leq C\left\|F_{B}\right\|_{-\frac{3}{2}}^{\Gamma} .
$$

Proof. Note that, according to (1.3) and (1.4),

$$
\int_{\Gamma} v \cdot\left(\lambda_{2} \otimes n\right) d \Gamma=\int_{\Gamma} \lambda_{2} \cdot(n \times v) d \Gamma=\lambda_{2} \cdot \int_{\Omega} \operatorname{rot} v d x=\lambda_{2}^{T} \varphi_{2}(v) .
$$

Using this and (2.3) we find from $\widetilde{L}[u p \lambda]^{T}=\left[F_{I} g F_{B} h_{1} h_{2}\right]^{T}$ the variational formulation

$$
\begin{aligned}
a(u, v) & -\int_{\Omega} p \operatorname{div} v d x+\lambda_{1}^{T} \varphi_{1}(v)+\lambda_{2}^{T} \varphi_{2}(v) \\
& =\int_{\Omega}\left(F_{I}+\nabla g\right) \cdot v d x+\int_{\Gamma} F_{B} \cdot v d \Gamma \\
& \quad \text { for all } v \in\left(H^{1}(\Omega)\right)^{N} \\
-\operatorname{div} u & =g \\
\varphi_{1}(u) & =h_{1} \\
\varphi_{2}(u) & =h_{2} .
\end{aligned}
$$

Lemma 1 yields that this problem has a unique solution $[u p \lambda]^{T} \in X$, and from the fact that $a(u, \cdot)$ and div vanish on $V_{0}$ we find

$$
\lambda_{i j}=\int_{\Omega}\left(F_{I}+\nabla g\right) \cdot v_{i j} d x+\int_{\Gamma} F_{B} \cdot v_{i j} d \Gamma
$$


where $i=1,2, \lambda_{i j}$ is the $j$-th component of $\lambda_{i}$, and the $v_{i j}$ form the dual basis of $V_{0}$ with respect to the $\varphi_{i}$, i.e. we have $\varphi_{i j}\left(v_{k l}\right)=\delta_{i k} \delta_{j l}$. All $v_{i j}$ are smooth, hence

$$
\|\lambda\|_{\mathbb{R}^{N} \times \mathbb{R}^{(N)}} \leq C\left(\left\|F_{I}\right\|_{9-2}^{\Omega}+\|g\|_{s-1}^{\Omega}+\left\|F_{B}\right\|_{9-\frac{3}{2}}^{\Gamma}\right)
$$

and (2.4) follow.

Let $s$ be integer for the moment, the general result will finally follow by interpolation. We will determine $u$ and $p$ by setting

$$
\begin{aligned}
& u=u_{0}+u_{1}+u_{2} \\
& p=p_{1}+p_{2}
\end{aligned}
$$

where

$$
\left.\begin{array}{rlrl}
-\Delta u_{1}+\nabla p_{1} & =F_{I}-\lambda_{1} \\
-\operatorname{div} u_{1} & =g
\end{array}\right\} \quad \text { in } \Omega
$$

$u_{0} \in V_{0}$ such that $\varphi_{i}\left(u_{0}\right)=-\varphi_{i}\left(u_{1}\right)+h_{i}(i=1,2)$ and

$$
\begin{aligned}
& \left.\begin{array}{rl}
-\Delta u_{2}+\nabla p_{2} & =0 \\
-\operatorname{div} u_{2} & =0
\end{array}\right\} \quad \text { in } \Omega \\
& \mathcal{T}\left(u_{2}, p_{2}\right) n=-\mathcal{T}\left(u_{1}, p_{1}\right) n+F_{B}-\lambda_{2} \times n=\Phi \text { on } \Gamma \\
& \varphi_{i}\left(u_{2}\right)=0 \quad(i=1,2) \text {. }
\end{aligned}
$$

Note that

$$
\int_{\Gamma} \Phi \cdot v=0 \quad \forall v \in V_{0}
$$

The regularity results for the Dirichlet problem of the Stokes equations yield that (2.7) has precisely one solution $\left(u_{1}, p_{1}\right) \in\left(H^{s}(\Omega)\right)^{N} \times H^{s-1}(\Omega)$ with $\int_{\Omega} p_{1} d x=0$ and an estimate

$$
\left\|u_{1}\right\|_{s}^{\Omega}+\left\|p_{1}\right\|_{s-1}^{\Omega} \leq C\left(\left\|F_{I}\right\|_{s-2}^{\Omega}+\left\|\lambda_{1}\right\|_{\mathbb{R}^{N}}+\|g\|_{s-1}^{\Omega}\right)
$$

holds [12: Theorem IV.6.1]. Thus we have $\Phi \in\left(H^{s-\frac{3}{2}}(\Gamma)\right)^{N}$ and

$$
\begin{aligned}
\|\Phi\|_{s-\frac{3}{2}}^{\Gamma} & \leq C\left(\left\|u_{1}\right\|_{s}^{\Omega}+\left\|p_{1}\right\|_{s-1}^{\Omega}+\left\|\lambda_{2}\right\|_{\left.\mathbb{R}^{(}{ }_{2}^{N}\right)}+\left\|F_{B}\right\|_{s-\frac{3}{2}}^{\Gamma}\right) \\
& \leq C\left(\left\|F_{I}\right\|_{s-2}^{\Omega}+\|g\|_{s-1}^{\Omega}+\|\lambda\|_{\left.\mathbb{R}^{N} \times \mathbb{R}^{(}{ }_{2}^{N}\right)}\right) .
\end{aligned}
$$

It remains to show that, for all $\Phi$ that satisfy (2.8), (2.9) has a unique solution $\left(u_{2}, p_{2}\right) \in\left(H^{s}(\Omega)\right)^{N} \times H^{s-1}(\Omega)$ satisfying an estimate

$$
\left\|u_{2}\right\|_{s}^{\Omega}+\left\|p_{2}\right\|_{s-1}^{\Omega} \leq C\|\Phi\|_{s-\frac{3}{2}}^{\Gamma} .
$$


From the discussion of the weak formulation we recall that (2.9) is a necessary condition for the solvability of $(2.8)$ and that the solution $\left(u_{2}, p_{2}\right)$ is unique. From a density argument it follows that we can assume $\Phi \in(C(\Gamma))^{N}$.

We will apply integral representations from the theory of hydrodynamic potentials. For the sake of brevity the description of the details will be restricted to the case $N=3$. For $x \in \Omega$ we use the ansatz

$$
\begin{aligned}
u_{2}(x) & =V(x, \psi) \\
V(x, \psi) & =\frac{1}{8 \pi} \int_{\Gamma}\left(\frac{I}{|x-y|}+\frac{(x-y)(x-y)^{T}}{|x-y|^{3}}\right) \psi(y) d \Gamma_{y} \\
p_{2}(x) & =\frac{1}{4 \pi} \int_{\Gamma} \frac{x-y}{|x-y|^{3}} \psi(y) d \Gamma_{y}
\end{aligned}
$$

where $\psi$ is a $\mathbb{R}^{3}$-valued (measurable) function on $\Gamma$. It is shown in [17: Chapter III] that $\left(u_{2}, p_{2}\right)$ satisfies the first three equations in (2.8) if $\psi$ is continuous and satisfies

$$
\left(\frac{1}{2} I+K\right) \psi=\Phi
$$

with

$$
(K \psi)(x)=-\frac{3}{4 \pi} \int_{\Gamma} \frac{(x-y)(x-y)^{T}}{|x-y|^{5}}(x-y) n(x) \psi(y) d \Gamma_{y} \quad(x \in \Gamma) .
$$

The operator $K$ is a weakly singular integral operator, hence it is compact on $\left(H^{0}(\Gamma)\right)^{N}$ and continuity of $\Phi$ implies continuity for all $\psi \in\left(H^{0}(\Gamma)\right)^{N}$ that satisfy (2.11) (see, e.g., [20: Theorems 12.1, 12.7 and 12.8]). Moreover, $K$ is a pseudodifferential operator [19], hence it is compact on $\left(H^{s-\frac{3}{2}}(\Gamma)\right)^{N}$ and therefore $\left(\frac{1}{2} I+K\right)$ is a Fredholm operator of index 0 on this space. Taking into account that $N\left(\frac{1}{2} I+K\right)$ consists of continuous functions one can conclude, using the results about the weak formulation, that $V(\cdot, \psi) \in$ $V_{0}$ for all $\psi \in N\left(\frac{1}{2} I+K\right)$. The mapping $\psi \mapsto V(\cdot, \psi)$ is injective [17], hence $\operatorname{dim} N\left(\frac{1}{2} I+\right.$ $K) \leq 6$. The necessary solvability conditions $(2.9)$ imply $\operatorname{codim} R\left(\frac{1}{2} I+K\right) \geq 6$, hence

$$
\operatorname{dim} N\left(\frac{1}{2} I+K\right)=\operatorname{codim} R\left(\frac{1}{2} I+K\right)=6,
$$

i.e. the solvability conditions (2.9) are also sufficient and the mapping $\psi \mapsto V(\cdot, \psi)$ maps $N\left(\frac{1}{2} I+K\right)$ onto $V_{0}$. Thus we can conclude that $(2.11)$ has precisely one solution such that $\varphi_{i}(V(\cdot, \psi))=0 \quad(i=1,2)$ satisfying an estimate

$$
\|\psi\|_{s-\frac{3}{2}}^{\Gamma} \leq C\|\Phi\|_{s-\frac{3}{2}}^{\Gamma}
$$

Finally we use the fact that the singular integral operator that maps $\psi$ to $\left.V(\cdot, \psi)\right|_{\Gamma}$ is a pseudodifferential operator of order -1 [19], hence we find that the trace of $u_{2}$ on $\Gamma$ is in $\left(H^{s-\frac{1}{2}}(\Gamma)\right)^{N}$ and

$$
\left\|u_{2}\right\|_{s-\frac{1}{2}}^{\Gamma} \leq C\|\Psi\|_{s-\frac{3}{2}}^{\Gamma} \leq C\|\Phi\|_{s-\frac{3}{2}}^{\Gamma} .
$$

The proof of (2.10) is completed now by another application of the regularity result on the Dirichlet problem. 
To show (2.5), consider the "adjoint" problem

$$
\left.\begin{array}{rl}
-\Delta v+\nabla q & =u \\
\operatorname{div} v & =0
\end{array}\right\} \quad \text { in } \Omega
$$

with $\mu \in \mathbb{R}^{\left(\begin{array}{c}N \\ 2\end{array}\right)}$ given by

$$
\mu_{j}=\int_{\Omega} u \cdot v_{2, j} d x
$$

which implies $\|\mu\|_{\left.\mathbb{R}^{(}{ }_{2}^{N}\right)} \leq C\|u\|_{0}^{\Omega}$. By examining the variational formulation of this problem in the same way as in Lemma 1 we find the existence of a weak solution of it that satisfies $\varphi_{1}(v)=0, \varphi_{2}(v)=0$. By the above regularity results we get

$$
\|v\|_{2}^{\Omega} \leq C\left(\|u\|_{0}^{\Omega}+\|\mu \otimes n\|_{\frac{1}{2}}^{\Gamma}\right) \leq C\|u\|_{0}^{\Omega}
$$

With this, we find by the second Green formula for the Stokes equations

$$
\begin{aligned}
\|u\|_{0}^{\Omega^{2}} & =(u,-\Delta v+\nabla q)_{0}+\int_{\Gamma}(\mathcal{T}(u, p) n \cdot v-\mathcal{T}(v, q) n \cdot u) d \Gamma \\
& =\int_{\Gamma}\left(F_{B} \cdot v+\mu \otimes n\right) d \Gamma=\int_{\Gamma} F_{B} \cdot v d \Gamma-\mu \cdot \varphi_{2}(u) \\
& \leq C\left\|F_{B}\right\|_{-\frac{3}{2}}^{\Gamma}\|v\|_{\frac{3}{2}}^{\Gamma} \leq C\left\|F_{B}\right\|_{-\frac{3}{2}}^{\Gamma}\|v\|_{2}^{\Omega} \leq C\left\|F_{B}\right\|_{-\frac{3}{2}}^{\Gamma}\|u\|_{0}^{\Omega}
\end{aligned}
$$

which proves (2.5)

\section{Perturbations of the domain and analytic expansions}

In order to describe the evolution of the domain we consider a fixed domain $\Omega_{0}$ which is supposed to be bounded, smooth, and locally on one side of its boundary $\Gamma_{0}$. Its outer normal vector will be denoted by $n$, and we choose a fixed vector-valued function $\zeta \in\left(C^{\infty}\left(\Gamma_{0}\right)\right)^{N}$ such that

$$
\gamma(\xi)=\zeta(\xi) \cdot n(\xi)>0 \quad \forall \xi \in \Gamma_{0}
$$

and a fixed constant $s_{0}>3+\frac{N-1}{2}$.

Lemma 3.1 (Description of perturbed domains). There is a $\delta_{0}>0$ such that for all $r \in B_{0}\left(\delta_{0}, H^{s_{0}}\left(\Gamma_{0}\right)\right)$ the following holds:

(i) The set

$$
\Gamma_{r}=\left\{\xi+\zeta(\xi) r(\xi) \mid \xi \in \Gamma_{0}\right\}
$$

is the boundary of a simply connected domain $\Omega_{r}$. 
(ii) There is a global diffeomorphism $z=z(r)$ mapping $\Omega_{0}$ onto $\Omega_{r}$ such that $z \in$ $\left(H^{s_{0}+\frac{1}{2}}\left(\Omega_{0}\right)\right)^{N}$ and

$$
\|z-\mathrm{id}\|_{s_{0}+\frac{1}{2}}^{\Omega_{0}} \leq C\|r\|_{s_{0}}^{\Gamma_{0}}
$$

with $C$ independent of $r$.

Proof. Statement (i): The collar manifold theorem implies the existence of a diffeomorphism between $I \times \Gamma_{0}$ and an open neighbourhood of $\Gamma_{0}$ in $\mathbb{R}^{N}$ where $I$ is a certain open neighbourhood of 0 in $\mathbb{R}$. The assertion follows thus from the embedding $H^{s_{0}}\left(\Gamma_{0}\right) \hookrightarrow C^{0}\left(\Gamma_{0}\right)$.

Statement (ii): We construct $z$ by setting $z=\operatorname{Tr}^{-1}(r \zeta)+$ id where $\operatorname{Tr}^{-1}$ is a fixed right inverse of the trace operator $\operatorname{Tr}$ from $H^{s_{0}+\frac{1}{2}}\left(\Omega_{0}\right)$ to $H^{s_{0}}\left(\Gamma_{0}\right)$. The embedding theorems yield then that $\|z-\mathrm{id}\|_{\left(C^{2}\left(\Omega_{0}\right)\right)^{N}}$ is small which implies the global injectivity of $z$. (For details see [13].)

Consider now, with the notation of the previous section, $s=s_{0}-\frac{1}{2}$, and $\Omega=\Omega_{r}$ the equations

$$
\begin{aligned}
& L[U P \Lambda]^{T}=[f 0]^{T}
\end{aligned}
$$

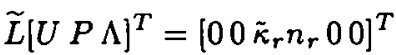

with $f \in\left(\left(H^{1}\left(\Omega_{r}\right)\right)^{N}\right)^{\prime}$ defined by

$$
f(v)=\int_{\Gamma_{r}} \tilde{\kappa}_{r} n_{r} \cdot v d \Gamma_{r}
$$

where $\tilde{\kappa}_{r}$ and $n_{r}$ are the double mean curvature and the outer normal vector of $\Gamma_{r}$, respectively. Using $r$ and $z(r)$ it is possible by means of Lemma 3 to transform both equations to $\Omega_{0}$, and in the sequel we will consider the operators $L, \widetilde{L}$ etc. as acting on function spaces defined on $\Omega_{0}$ and depending on $r \in B_{0}\left(\delta_{0}, H^{s_{0}}\left(\Gamma_{0}\right)\right)$. Thus we get

$$
\begin{aligned}
L(r)[u(r) p(r) \lambda(r)]^{T} & =F(r) \\
\widetilde{L}(r)[u(r) p(r) \lambda(r)]^{T} & =\widetilde{F}(r)
\end{aligned}
$$

with

$$
\begin{aligned}
{[u(r) p(r) \lambda(r)]^{T} } & =[U \circ z(r) P \circ z(r) \Lambda]^{T} \\
L(r)\left[\begin{array}{l}
u \\
p \\
\lambda
\end{array}\right] & =\left[\begin{array}{c}
A(r) u+(B(r))^{\prime}\left[\begin{array}{l}
p \\
\lambda
\end{array}\right] \\
B(r) u
\end{array}\right] \\
F(r) & =[f(r) 0]^{T} \\
\widetilde{F}(r) & =\left[00 \kappa_{r} \nu(r) 00\right]^{T} \\
(A(r) u) v & =\int_{\Omega_{0}} \sum_{i, j, m, l=1}^{N}\left(a^{m j} \frac{\partial u_{i}}{\partial x_{m}}+a^{m i} \frac{\partial u_{j}}{\partial x_{m}}\right)\left(a^{l j} \frac{\partial v_{i}}{\partial x_{l}}+a^{l i} \frac{\partial v_{j}}{\partial x_{l}}\right) \operatorname{det} \mathcal{A} d x
\end{aligned}
$$




$$
\begin{aligned}
& B(r) u=\left[\begin{array}{c}
-\operatorname{div}_{r} \operatorname{det} \mathcal{A} \\
\int_{\Omega_{0}} u \operatorname{det} \mathcal{A} d x \\
\int_{\Omega_{0}} \operatorname{rot}_{r} \operatorname{det} \mathcal{A} d x
\end{array}\right] \\
& f(r) v=\int_{\Gamma} \chi_{r} \kappa_{r} \nu(r) \cdot v d \Gamma_{0} \\
& \tilde{L}(r)[u p \lambda]=\left[\begin{array}{c}
-\Delta_{r} u+\nabla_{r} p+\lambda_{1} \\
-\operatorname{div}_{r} u \\
\mathcal{T}_{r}(u, p) \nu(r)+\lambda_{2} \otimes \nu(r) \\
\int_{\Omega_{0}} u \operatorname{det} \mathcal{A} d x \\
\int_{\Omega_{0}} \operatorname{rot}_{r} u \operatorname{det} \mathcal{A} d x
\end{array}\right] \\
& \left(\nabla_{r} p\right)_{i}=\sum_{j} a^{j i} \frac{\partial p}{\partial x_{j}} \\
& \operatorname{div}_{r} u=\sum_{i, j} a^{j i} \frac{\partial u_{i}}{\partial x_{j}} \\
& \left(\operatorname{rot}_{r} u\right)_{K(i, j)}=\sum_{l}\left(a^{l i} \frac{\partial u_{j}}{\partial x_{l}}-a^{l j} \frac{\partial u_{i}}{\partial x_{l}}\right) \\
& \left(\Delta_{r} u\right)_{i}=\sum_{j, k, l} a^{j l} \frac{\partial}{\partial x_{j}}\left(a^{k l} \frac{\partial u_{i}}{\partial x_{k}}\right) \\
& (\nabla, u)_{i j}=\sum_{k} a^{k j} \frac{\partial u_{i}}{\partial x_{k}} \\
& \mathcal{T}_{r}(u, p)=\left(\nabla_{r} u\right)+\left(\nabla_{r} u\right)^{T}-p I
\end{aligned}
$$

where $v \in\left(H^{1}\left(\Omega_{0}\right)\right)^{N}, \mathcal{A}$ is the Jacobian $\frac{\partial z(r)}{\partial \xi}, a^{i j}$ are the elements of $\mathcal{A}^{-1}, \kappa_{r}=$ $\tilde{\kappa}_{r} \circ z(r), \nu(r)=n_{r} \circ z(r)$, and $\chi_{r}$ is a scalar function on $\Gamma_{0}$ describing the "change of the surface element" when $\Gamma_{0}$ is mapped to $\Gamma_{r}$ by $z$.

Let $E$ and $F$ be Banach spaces. An operator $T$ that maps a neighbourhood of $x_{0} \in E$ to $F$ is called analytic near $x_{0}$ if it has a series representation

$$
T(x)=T_{0}+\sum_{k=1}^{\infty} T_{k}\left(x-x_{0}, \ldots, x-x_{0}\right)
$$

with symmetric $k$-linear operators $T_{k}$ and positive convergence radius. We will use the well-known facts that the sum, the composition and, if $F$ is a Banach algebra, the (pointwise) product of (locally) analytic operators is (locally) analytic.

Let $\mathcal{I}$ denote the embedding operator of $\tilde{X}$ into $X$.

Lemma 4 (Analyticity of the perturbation).

(i) The operators $L, \widetilde{L}, \dot{F}$, and $\widetilde{F}$ are analytic near 0 as functions of $r \in H^{s_{0}}\left(\Gamma_{0}\right)$ into $\mathcal{L}(X, Y), \mathcal{L}(\tilde{X}, \tilde{Y}), Y$, and $\tilde{Y}$, respectively. 
(ii) The estimates

$$
\begin{aligned}
&\left\|F_{k}\left(r_{1}, \ldots, r_{k}\right)\right\|_{Y} \leq C_{k}\left\|r_{1}\right\|_{s_{0}}^{\Gamma_{0}} \cdots\left\|r_{k-1}\right\|_{s_{0}}^{\Gamma_{0}}\left\|r_{k}\right\|_{\frac{3}{2}}^{\Gamma_{0}} \\
&\left\|L_{k}\left(r_{1}, \ldots, r_{k}\right) \mathcal{I}\right\|_{\mathcal{L}(\tilde{X}, Y)} \leq C_{k}\left\|r_{1}\right\|_{s_{0}}^{\Gamma_{0}} \cdots\left\|r_{k-1}\right\|_{s_{0}}^{\Gamma_{0}}\left\|r_{k}\right\|_{\frac{1}{2}}^{\Gamma_{0}}
\end{aligned}
$$

hold.

Proof. We have, writing $\tilde{r}=\operatorname{Tr}^{-1}(r \zeta)$,

$$
\|\mathcal{A}-I\|_{\left(C^{0}\left(\Omega_{0}\right)^{N \times N)}\right.}=\left\|\frac{\partial \tilde{r}}{\partial x}\right\|_{\left(C^{0}\left(\Omega_{0}\right)^{N \times N}\right)} \leq C\|r\|_{s_{0}^{0}}^{\Gamma_{0}}
$$

hence the inverse of $\mathcal{A}$ exists for all $x \in \Omega_{0}$ and can be represented by a Neumann series if $\|r\|_{s_{0}}^{\Gamma_{0}}$ is sufficiently small. Thus we get a series representation

$$
a^{i j}=\delta_{i j}+\sum_{k=1}^{\infty} a_{k}^{i j}(r, \ldots, r)
$$

where the $a_{k}^{i j}$ are linear combinations of first partial derivatives of components of $\tilde{r}$. $\operatorname{det} \mathcal{A}$ can also be written as a (finite) series of this type, and thus, for arbitrary $u, v \in H^{1}\left(\Omega_{0}\right)$, $\left(A_{k}\left(r_{1}, \ldots, r_{k}\right) u\right) v$ is a linear combination of terms

$$
\int_{\Omega} \frac{\partial u_{i}}{\partial x_{m}} \frac{\partial v_{j}}{\partial x_{l}} \prod_{\sigma=1}^{k} \frac{\partial\left(\tilde{r}_{\sigma}\right)_{t_{\sigma}}}{\partial x_{\tau_{\sigma}}} d x
$$

with $\tilde{r}_{\sigma}=\operatorname{Tr}^{-1}\left(r_{\sigma} \zeta\right)$.

We will apply to these terms the estimate

$$
\left|\int_{\Omega} \psi_{1} \psi_{2} \psi_{3} d x\right| \leq\left|\int_{\Omega} \psi_{1} \psi_{2} d x\right|\left\|\psi_{3}\right\|_{C^{0}\left(\Omega_{0}\right)} \leq C\left\|\psi_{1}\right\|_{0}^{\Omega_{0}}\left\|\psi_{2}\right\|_{0}^{\Omega_{0}}\left\|\psi_{3}\right\|_{s_{0}-2}^{\Omega_{0}}
$$

holding for all $\psi_{1}, \psi_{2} \in H^{0}\left(\Omega_{0}\right)$ and $\psi_{3} \in H^{s_{0}-2}\left(\Omega_{0}\right)$. If we set

$$
\psi_{1}=\frac{\partial u_{i}}{\partial x_{m}}, \quad \psi_{2}=\frac{\partial v_{j}}{\partial x_{l}}, \quad \psi_{3}=\prod_{\sigma=1}^{k} \frac{\partial\left(\tilde{r}_{\sigma}\right)_{t_{\sigma}}}{\partial x_{\tau_{\sigma}}}
$$

and take into account that $H^{s_{0}-2}\left(\Omega_{0}\right)$ is a Banach algebra we obtain after summation that $A$ is analytic near 0 as a function of $r \in H^{s_{0}}\left(\Gamma_{0}\right)$ into $\mathcal{L}\left(\left(H^{1}\left(\Omega_{0}\right)\right)^{N},\left(\left(H^{1}\left(\Omega_{0}\right)\right)^{N}\right)^{\prime}\right)$. If we assume $u \in H^{s_{0}-\frac{1}{2}}\left(\Omega_{0}\right)$ and set

$$
\psi_{1}=\frac{\partial v_{j}}{\partial x_{l}}, \quad \psi_{2}=\frac{\partial\left(\tilde{r}_{k}\right)_{t_{k}}}{\partial x_{\tau_{k}}}, \quad \psi_{3}=\frac{\partial u_{i}}{\partial x_{m}} \prod_{\sigma=1}^{k-1} \frac{\partial\left(\tilde{r}_{\sigma}\right)_{t_{o}}}{\partial x_{\tau_{\sigma}}}
$$


we get by the same arguments

$$
\left\|A_{k}\left(r_{1}, \ldots, r_{k}\right) u\right\|_{\left(\left(H^{1}\left(\Omega_{0}\right)\right)^{N}\right)^{\prime}} \leq C_{k}\|u\|_{s_{0}-\frac{1}{2}}^{\Omega_{0}}\left\|r_{1}\right\|_{s_{0}}^{\Gamma_{0}} \cdots\left\|r_{k-1}\right\|_{s_{0}}^{\Gamma_{0}}\left\|r_{k}\right\|_{\frac{1}{2}}^{\Gamma_{0}} .
$$

Together with analogous considerations on $B, B^{\prime}$, and the integrals describing the auxiliary conditions this yields the assertions on $L$.

We introduce regular smooth local parametrizations of $\Gamma_{0}=\bigcup_{j=1}^{J} \Gamma_{0}^{(j)}$ by

$$
\Gamma_{0}^{(j)}: \quad \xi=\xi^{(j)}(w) \quad\left(w \in W_{j} \subset \mathbb{R}^{N-1}\right)
$$

which yield local parametrizations of $\Gamma_{r}=\bigcup_{j=1}^{J} \Gamma_{r}^{(j)}$ by

$$
\Gamma_{r}^{(j)}: \quad x=x_{r}^{(j)}(w)=\xi^{(j)}(w)+\left(r\left(\xi^{(j)}(w)\right) \zeta\left(\xi^{(j)}(w)\right)\right.
$$

whose regularity follows for small $\|r\|_{g_{0}}^{\Gamma_{0}}$ from the regularity of the $\xi^{(j)}$. On the $j$-th coordinate patch, $\nu(r)$ can be characterized by the equations

$$
\begin{aligned}
\nu(r)=\frac{\tilde{\nu}(r)}{|\tilde{\nu}(r)|} & =\frac{\tilde{\nu}(r)}{\sqrt{\tilde{\nu}(r)^{T} \tilde{\nu}(r)}} \\
\left(\frac{\partial x_{r}^{(j)}}{\partial w}\right)^{T} \tilde{\nu}(r) & =0 \\
n \cdot \tilde{\nu}(r) & =1 .
\end{aligned}
$$

The latter two of these equations form a system of $N$ scalar linear equations for $\tilde{\nu}(r)$ which at $r=0$ has the unique solution $\tilde{\nu}(0)=\nu(0)=n$. Inverting this system for small $\|r\|_{s_{0}}^{\Gamma_{0}}$ using the same arguments as above and taking into account that $|\tilde{\nu}(r)|$ is near 1 for small $r$ we get a convergent series representation

$$
\nu(r) \circ \xi^{(j)}=n \circ \xi^{(j)}+\sum_{k=1}^{\infty} \nu_{k}^{(j)}(r, \ldots, r)
$$

where the $\nu_{k}^{(j)}\left(r_{1}, \ldots, r_{k}\right)$ are sums of products of smooth functions with the $\left.\left(r_{\sigma} \circ \xi^{(j}\right)\right)$ or their first partial derivatives. Hence $\nu$ is an analytic mapping near 0 from $H^{s_{0}}\left(\Gamma_{0}\right)$ to $\left(H^{s_{0}-2}\left(\Gamma_{0}\right)\right)^{N}$.

Moreover, we have in local coordinates on $\Gamma_{0}^{(j)}$

$$
\begin{gathered}
\chi_{r}=\frac{\sqrt{g_{r}^{(j)}}}{\sqrt{g_{0}^{(j)}}} \\
\kappa_{r} \nu(r)=\Delta_{\Gamma_{r}} x_{r}=\frac{1}{\sqrt{g_{r}^{(j)}}} \sum_{i, k=1}^{N-1} \frac{\partial}{\partial w_{i}}\left(g_{(j)}^{i k} \frac{\partial x_{r}^{(j)}}{\partial w_{k}}\right)
\end{gathered}
$$


with

$$
g_{r}^{(j)}=\operatorname{det} G_{r}^{(j)}, \quad g_{(j)}^{i k}=\left[G_{r}^{(j)}\right]_{i k}, \quad G_{r}^{(j)}=\left(\frac{\partial x_{r}^{(j)}}{\partial w}\right)^{T}\left(\frac{\partial x_{r}^{(j)}}{\partial w}\right)
$$

and we find by analogous arguments that the mappings $r \mapsto \chi_{r}$ and $r \mapsto \kappa_{r}$ are analytic near 0 from $H^{s_{0}}\left(\Gamma_{0}\right)$ to $H^{s_{0}-2}\left(\Gamma_{0}\right)$. Thus we get the assertions on $F$ and $\widetilde{F}$.

The analytic dependence of $\tilde{L}$ on $r \in H^{s_{0}}\left(\Gamma_{0}\right)$ follows from the above considerations and the Banach algebra properties of the spaces $H^{s_{0}-\frac{5}{2}}\left(\Omega_{0}\right), H^{s_{0}-\frac{3}{2}}\left(\Omega_{0}\right)$, and $H^{s_{0}-2}\left(\Gamma_{0}\right)$

Lemma 5 (Analytic dependence of the solution). Let $u(r)$ be defined by (3.2). The mapping $r \mapsto u(r)$ is well-defined on $B_{0}\left(\varepsilon, H^{s_{0}}\left(\Gamma_{0}\right)\right)$ for some $\varepsilon>0$ into $\left(H^{s_{0}-\frac{1}{2}}\left(\Omega_{0}\right)\right)^{N}$. It is analytic near 0 and estimates

$$
\left\|u_{k}\left(r_{1}, \ldots, r_{k}\right)\right\|_{1}^{\Omega_{0}} \leq C_{k}\left\|r_{1}\right\|_{s_{0}}^{\Gamma_{0}} \ldots\left\|r_{k-1}\right\|_{s_{0}}^{\Gamma_{0}}\left\|r_{k}\right\|_{\frac{3}{2}}^{\Gamma_{0}}
$$

hold.

Proof. Writing $\underline{v}(r)=[u(r) p(r) \lambda(r)]^{T} \in \tilde{X}$ we have, due to Lemmas $2 /(\mathrm{i})$ and Lemma $4 /(i)$ by the real-analytic version of the Implicit Function Theorem that the mapping $r \mapsto \underline{v}(r)$ exists and is analytic near 0 from $H^{s_{0}}\left(\Gamma_{0}\right)$ to $\tilde{X}$, with estimates

$$
\left\|\underline{v}_{k}\left(r_{1}, \ldots, r_{k}\right)\right\|_{\widetilde{X}} \leq C_{k}\left\|r_{1}\right\|_{s_{0}}^{\Gamma_{0}} \ldots\left\|r_{k}\right\|_{s_{0}}^{\Gamma_{0}} .
$$

The assertions on the mapping $r \mapsto u(r)$ follow immediately from this.

To prove (3.4) it is sufficient to establish

$$
\left\|\underline{v}_{j}\left(r_{1}, \ldots, r_{j}\right)\right\|_{X} \leq C_{j}\left\|r_{1}\right\|_{s_{0}}^{\Gamma_{0}} \ldots\left\|r_{j-1}\right\|_{s_{0}}^{\Gamma_{0}}\left\|r_{j}\right\|_{\frac{3}{2}}^{\Gamma_{0}}
$$

This will be done by induction. For $j=1$, we have

$$
\begin{aligned}
\left\|\underline{v}_{1}(r)\right\|_{X} & =\left\|L(0)^{-1} F_{1}(r)-L(0)^{-1} L_{1}(r) \underline{v}_{0}\right\|_{X} \\
& \leq C\left(\left\|F_{1}(r)\right\|_{Y}+\left\|L_{1}(r) I\right\|_{\mathcal{L}(\widetilde{X}, Y)}\right) \\
& \leq C\|r\|_{\frac{3}{2}}^{\Gamma_{0}}
\end{aligned}
$$

where Lemma 4/(ii) has been used. Suppose now (3.6) holds for all $j \leq k-1$. Taking the $k$-th Fréchet derivative at $r=0$ on both sides of the equation $L(r) \underline{v}(r)=F(r)$ and applying $L(0)^{-1}$ yields

$$
\begin{aligned}
\underline{v}_{k}\left(r_{1}, \ldots, r_{k}\right)= & L(0)^{-1}\left(F_{k}\left(r_{1}, \ldots, r_{k}\right)\right. \\
& \left.-\sum_{j=1}^{k} \frac{1}{j !(k-j) !} \sum_{\pi} L_{j}\left(r_{\pi(1)}, \ldots, r_{\pi(j)}\right) \underline{v}_{k-j}\left(r_{\pi(j+1)}, \ldots, r_{\pi(k)}\right)\right)
\end{aligned}
$$


where $\pi$ runs over all permutations of $\{1, \ldots, k\}$. We will estimate the terms on the right separately, using Lemma $4 /(i i)$. Thus we get

$$
\left\|L(0)^{-1} F_{k}\left(r_{1}, \ldots, r_{k}\right)\right\|_{X} \leq C\left\|F_{k}\left(r_{1}, \ldots, r_{k}\right)\right\|_{Y} \leq C_{k}\left\|r_{1}\right\|_{s_{0}}^{\Gamma_{0}} \cdots\left\|r_{k-1}\right\|_{s_{0}}^{\Gamma_{0}}\left\|r_{k}\right\|_{\frac{3}{2}}^{\Gamma_{0}},
$$

for the terms in the sum over $j$ with $\pi^{-1}(k) \leq j$ we find. using (3.5),

$$
\begin{aligned}
\| L(0)^{-1} & L_{j}\left(r_{\pi(1)}, \ldots, r_{\pi(j)}\right) \underline{v}_{k-j}\left(r_{\pi(j+1)}, \ldots, r_{\pi(k)}\right) \|_{X} \\
& \leq C\left\|L_{j}\left(r_{\pi(1)}, \ldots, r_{\pi(j)}\right) \mathcal{I}\right\|_{\mathcal{L}(\widetilde{X}, Y)}\left\|\underline{v}_{k-j}\left(r_{\pi(j+1)}, \ldots, r_{\pi(k)}\right)\right\|_{\tilde{X}} \\
& \leq C_{k}\left\|r_{1}\right\|_{s_{0}}^{\Gamma_{0}} \cdots\left\|r_{k-1}\right\|_{s_{0}}^{\Gamma_{0}}\left\|r_{k}\right\|_{\frac{1}{2}}^{\Gamma_{0}},
\end{aligned}
$$

and for the other terms, using the induction assumption,

$$
\begin{aligned}
\| L(0)^{-1} & L_{j}\left(r_{\pi(1)}, \ldots, r_{\pi(j)}\right) \underline{v}_{k-j}\left(r_{\pi(j+1)}, \ldots, r_{\pi(k)}\right) \|_{X} \\
& \leq C\left\|L_{j}\left(r_{\pi(1)}, \ldots, r_{\pi(j)}\right)\right\|_{\mathcal{L}(X, Y)}\left\|\underline{v}_{k-j}\left(r_{\pi(j+1)}, \ldots, r_{\pi(k)}\right)\right\|_{X} \\
& \leq C_{k}\left\|r_{1}\right\|_{s_{0}}^{\Gamma_{0}} \ldots\left\|r_{k-1}\right\|_{s_{0}}^{\Gamma_{0}}\left\|r_{k}\right\|_{\frac{3}{2}}^{\Gamma_{0}}
\end{aligned}
$$

Hence (3.6) holds also for $j=k$

We describe now the moving boundary $\Gamma(t)$ near $\Gamma_{0}$ by

$$
\Gamma(t)=\Gamma_{r(t)}
$$

The kinematic boundary condition takes then the form

$$
\frac{\partial r}{\partial t}=\frac{\operatorname{Tr}_{\Gamma_{0}}(u(r)) \cdot \nu(r)}{\zeta \cdot \nu(r)}=\rho(r),
$$

i.e. our moving boundary problem is reformulated as a nonlinear nonlocal evolution equation for $r$. Using the inequality

$$
\left\|\psi_{1} \psi_{2}\right\|_{\frac{1}{2}}^{\Gamma_{0}} \leq C\left\|\psi_{1}\right\|_{C^{1}\left(\Gamma_{0}\right)}\left\|\psi_{2}\right\|_{\frac{1}{2}}^{\Gamma_{0}} \leq C\left\|\psi_{1}\right\|_{s_{0}-1}^{\Gamma_{0}}\left\|\psi_{2}\right\|_{\frac{1}{2}}^{\Gamma_{0}}
$$

and the Banach algebra property of $H^{s_{0}-1}\left(\Gamma_{0}\right)$ we find by arguments similar to the ones given above that $\rho$ is analytic near 0 from $H^{s_{0}}\left(\Gamma_{0}\right)$ to $H^{s_{0}-1}\left(\Gamma_{0}\right)$ and we have additional estimates

$$
\left\|\rho_{k}\left(r_{1}, \ldots, r_{k}\right)\right\|_{\frac{1}{2}}^{\Gamma_{0}} \leq C_{k}\left\|r_{1}\right\|_{s_{0}}^{\Gamma_{0}} \cdots\left\|r_{k-1}\right\|_{s_{0}}^{\Gamma_{0}}\left\|r_{k}\right\|_{\frac{3}{2}}^{\Gamma_{0}} .
$$

Note that in all estimates for $k$-linear forms the constants $C_{k}$ can be chosen such that

$$
C_{k} \sim O\left(M^{k}\right)
$$




\section{A chain rule}

In the following we suppose additionally that $\Omega_{0}$ is strictly star-shaped, i.e. there is a smooth positive real-valued function $R_{0}$ defined on the unit sphere $S^{N-1}$ such that (after a suitable translation)

$$
\Gamma_{0}=\left\{\theta R_{0}(\theta) \mid \theta \in S^{N-1}\right\}
$$

Note that the mapping $\Phi_{0}: S^{N-1} \longrightarrow \Gamma_{0}$ defined by $\Phi_{0}(\theta)=\zeta R_{0}(\theta)$ is a $C^{\infty}$. diffeomorphism between $S^{N-1}$ and $\Gamma_{0}$, hence the direct image map $\Phi_{0}^{*}$ defined by $\left(\Phi_{0}^{*} \varphi\right)(\theta)=\varphi\left(\Phi_{0}(\theta)\right)$ is an isomorphism from $C^{\infty}\left(\Gamma_{0}\right)$ to $C^{\infty}\left(S^{N-1}\right)$ and from $H^{s_{0}}\left(\Gamma_{0}\right)$ to $H^{s_{0}}\left(S^{N-1}\right)$.

We choose $\zeta(\xi)=\frac{\xi}{|\xi|}$ and consider a fixed system $\left\{Q_{j} \mid j=1, \ldots,\left(\begin{array}{c}N \\ 2\end{array}\right)\right\}$ of linear independent skew-symmetric $(N \times N)$-matrices. We introduce on $S^{N-1}$ and $\Gamma_{0}$, respectively, the first order linear differential operators $\widetilde{D}_{j}$ and $D_{j}$ by

$$
\begin{aligned}
& \widetilde{D}_{j} \psi(\zeta)=\left.\frac{d}{d \tau}\left(\left(\psi \circ \exp ^{\tau Q_{j}}\right)(\zeta)\right)\right|_{\tau=0} \\
& D_{j} \varphi(\xi)=\left.\frac{d}{d \tau}\left(\left(\varphi \circ \Phi_{0} \circ \exp ^{\tau Q_{j}} \circ \Phi_{0}^{-1}\right)(\xi)\right)\right|_{\tau=0}
\end{aligned}
$$

and for multiindices $\alpha=\left(\alpha_{1} \ldots \alpha_{\left(\begin{array}{c}N \\ 2\end{array}\right)}\right)$ we set $\left.\left.D^{\alpha}=D_{1}^{\alpha_{1}} \ldots D_{\left(\begin{array}{c}N \\ 2\end{array}\right)}^{\alpha}\right)^{N}\right), \widetilde{D}^{\alpha}$ is defined analogously.

In the following, $T^{(k)}(x)[\cdot, \ldots, \cdot]$ will denote the $k$-th Fréchet derivative of $T$ at $x$.

Lemma 6 (Chain rule). Assume $r$ to be smooth and $\|r\|_{s_{0}}^{\Gamma_{0}}$ sufficiently small. Then

$$
D^{\alpha}(\rho(r))=\sum_{k=1}^{|\alpha|} \sum_{\beta_{1}+\ldots+\beta_{k}=\alpha} C_{\beta_{1}, \ldots, \beta_{k}} \rho^{(k)}(r)\left[D^{\beta_{1}}\left(r+\mathcal{R}_{0}\right), \ldots, D^{\beta_{k}}\left(r+\mathcal{R}_{0}\right)\right]
$$

where $\mathcal{R}_{0}=\Phi_{0}^{*-1} R_{0}$, all occurring $\beta_{l}$ are non-zero, and for $k=1$ we have $C_{\alpha}=1$.

Proof. Define the operators $\tilde{\rho}, \tilde{u}$, and $\tilde{\nu}$ acting on the smooth functions in a small ball around $R_{0}$ in $H^{s_{0}}\left(S^{N-1}\right)$ by

$$
\tilde{\rho}(R)=\rho\left(\Phi_{0}^{*-1}\left(R-R_{0}\right)\right)
$$

and

$$
\begin{aligned}
& \tilde{u}(R)=u\left(\Phi_{0}^{*-1}\left(R-R_{0}\right)\right) \\
& \tilde{\nu}(R)=\nu\left(\Phi_{0}^{*-1}\left(R-R_{0}\right)\right) .
\end{aligned}
$$

We will show the equality

$$
D^{\alpha} \tilde{\rho}(R)=\sum_{k=1}^{|\alpha|} \sum_{\beta_{1}+\ldots+\beta_{k}=\alpha} C_{\beta_{1}, \ldots, \beta_{k}} \tilde{\rho}^{(k)}(R)\left[\tilde{D}^{\beta_{1}} R, \ldots, \widetilde{D}^{\beta_{k}} R\right]
$$


with the same additional assertions as above. This is equivalent to the lemma because of

$$
\begin{aligned}
r & =\Phi_{0}^{*-1}\left(R-R_{0}\right) \\
\Phi_{0}^{*-1}\left(\widetilde{D}^{\alpha} \psi\right) & =D^{\alpha}\left(\Phi_{0}^{*-1} \psi\right) \\
\tilde{\rho}^{(k)}(R)\left[h_{1}, \ldots, h_{k}\right] & =\rho^{(k)}\left(\Phi_{0}^{*-1}\left(R-R_{0}\right)\right)\left[\Phi_{0}^{*-1} h_{1}, \ldots, \Phi_{0}^{*-1} h_{k}\right]
\end{aligned}
$$

where the last statement holds for all $h_{l} \in H^{s_{0}}\left(\Gamma_{0}\right)$ and is obtained by calculating the $k$-th Fréchet derivative of both sides of (4.2).

The proof of (4.3) will be given by induction over $|\alpha|$ and rests essentially on the invariance of the problem under rigid body motions, in particular, under rotations around the origin.

1. $|\alpha|=1$ : Choose a fixed $j \in\left\{1, \ldots,\left(\begin{array}{c}N \\ 2\end{array}\right)\right\}$ and consider the one-parameter family of rotations around the origin described by $x \mapsto \exp ^{\tau Q} ; x$ with $\tau$ varying in a small open interval containing 0 . Let $\widetilde{\Omega}_{R}$ be the bounded domain with boundary $\{\theta R(\theta) \mid \theta \in$ $\left.S^{N-1}\right\}$. Then clearly $\exp ^{\tau Q_{j}}\left[\widetilde{\Omega}_{R}\right]=\widetilde{\Omega}_{R^{r}}$ with

$$
R^{\tau}(\zeta)=R\left(e^{-\tau Q_{j}} \zeta\right)
$$

Taking into account now the fact that the boundary value problem (2.1) as well as the auxiliary conditions $\varphi_{1}(u)=0, \varphi_{2}(u)=0$ are invariant with respect to rotations, i.e. that the coordinate change $x \mapsto \exp ^{r Q ;} x$ does not alter the form of the equations for fixed $Q_{j}$, we find

$$
\tilde{u}\left(R^{\tau}\right)\left(\left(\Phi_{0} \circ \exp ^{\tau Q_{j}} \circ \Phi_{0}^{-1}\right)(\xi)\right)=\exp ^{\tau Q_{j}} \tilde{u}(R)(\xi)
$$

and further, using (3.7) and the fact that $\exp ^{\tau Q_{j}}$ is an orthogonal matrix,

$$
\begin{aligned}
\tilde{\nu}\left(R^{\tau}\right) & \left(\left(\Phi_{0} \circ \exp ^{\tau Q_{j}} \circ \Phi_{0}^{-1}\right)(\xi)\right) \\
\zeta\left(\left(\Phi_{0} \circ \exp ^{\tau Q_{j}} \circ \Phi_{0}^{-1}\right)(\xi)\right) & =\exp ^{\tau Q_{j}} \tilde{\nu}(R)(\xi) \\
\tilde{\rho}\left(R^{\tau}\right)\left(\left(\Phi_{0} \circ \exp ^{\tau Q_{j}} \circ \Phi_{0}^{-1}\right)(\xi)\right) & =\tilde{\rho}(R)(\xi)
\end{aligned}
$$

for all $\xi \in \Gamma_{0}$. Differentiating the last equation with respect to $\tau$ at $\tau=0$ yields

$$
D_{j} \tilde{\rho}(R)=\tilde{\rho}^{\prime}(R)[\widetilde{D}, R]
$$

which is (4.3) for $|\alpha|=1$.

Moreover, starting from (4.4), by induction one proves

$$
\begin{aligned}
D_{j} \tilde{\rho}^{(k)}(R)\left[h_{1}, \ldots, h_{k}\right]= & \sum_{l=1}^{k} \tilde{\rho}^{(k)}(R)\left[h_{1}, \ldots, h_{l-1}, \widetilde{D}_{j} h_{l}, h_{l+1}, \ldots, h_{k}\right] \\
& +\tilde{\rho}^{(k+1)}(R)\left[\tilde{D}_{j} R, h_{1}, \ldots, h_{k}\right]
\end{aligned}
$$

for all $k \in \mathbb{N}$ and $h_{1}, \ldots, h_{k} \in H^{s_{0}+1}\left(\Gamma_{0}\right)$ where the induction step only consists in calculating the Fréchet derivative.

2. Suppose now (4.3) holds for $\left|\alpha^{\prime}\right|=m$, consider $\alpha$ with $|\alpha|=m+1$. Writing $D^{\alpha} \tilde{\rho}(R)=D_{j} D^{\alpha^{\prime}} \tilde{\rho}(R)$, applying the induction assumption and (4.5), and rearranging the terms according to the order of the Fréchet derivative completes the proof 
Expansions of $\rho(r)$ and $\rho^{(k)}(r)$ in (4.1) and "comparison of coefficients" yields

$$
\begin{aligned}
& D^{\alpha} \rho_{m}\left(r^{(1)}, \ldots, r^{(m)}\right)=\frac{1}{m !} \\
& \quad \times \sum_{\pi} \sum_{l=0}^{m} \sum_{k=\max \{1, m-l\}}^{|\alpha|} \sum_{\beta_{1}+\ldots+\beta_{k}=\alpha} C_{\beta_{1}, \ldots, \beta_{k}} \frac{(k+l) !}{l !(m-l) !(k-m+l) !} \\
& \quad \times \sum_{\sigma} \rho_{k+l}\left(r^{(\pi(1))}, \ldots, r^{(\pi(l))}, D^{\beta_{\sigma(1)}(\pi(l+1))},\right. \\
& \quad \ldots, D^{\beta_{\sigma(m-l)}} r^{(\pi(m))}, D^{\beta_{\sigma(m-l+1)}} \mathcal{R}_{0}, \ldots, D^{\left.\beta_{\sigma(k)} \mathcal{R}_{0}\right)}
\end{aligned}
$$

where $\pi$ and $\sigma$ run over all permutations of $\{1, \ldots, m\}$ and $\{1, \ldots, k\}$, respectively. Considering the special case $m=1$ and using that $\left|\beta_{j}\right| \leq|\alpha|-1$ for $j=1, \ldots, k$ if $k \geq 2$ we can prove the commutator estimates

$$
\begin{gathered}
\left\|\left(D^{\alpha} \rho_{1}-\rho_{1} D^{\alpha}\right) r\right\|_{\frac{1}{2}}^{\Gamma_{0}} \leq C\|r\|_{|\alpha|+\frac{1}{2}}^{\Gamma_{0}} \\
\left\|\left(D^{\alpha} \rho_{1}-\rho_{1} D^{\alpha}\right) r\right\|_{s_{0}-1}^{\Gamma_{0}} \leq C\|r\|_{|\alpha|+s_{0}-1}^{\Gamma_{0}}
\end{gathered}
$$

In the sequel, let $s_{1}$ be the smallest integer such that $s_{1}>3+\frac{N-1}{2}$. Note that the vector fields on $\Gamma_{0}$ that correspond to the differential operators $D_{j}$ span the tangent space in any $\xi \in \Gamma_{0}$, therefore the bilinear forms

$$
\begin{array}{ll}
(\varphi, \psi)_{s}=\sum_{|\alpha| \leq s-1}\left(D^{\alpha} \varphi, D^{\alpha} \psi\right)_{1} \quad\left(s \leq s_{1}\right) \\
(\varphi, \psi)_{s}=\sum_{|\alpha| \leq s-s_{1}}\left(D^{\alpha} \varphi, D^{\alpha} \psi\right)_{s_{1}} & \left(s>s_{1}\right)
\end{array}
$$

with

$$
(\varphi, \psi)_{1}=\int_{\Gamma_{0}}\left(\varphi \psi+\nabla_{\Gamma_{0}} \varphi \cdot \nabla_{\Gamma_{0}} \psi\right) d \Gamma_{0}
$$

can and will be used as scalar products on $H^{s}\left(\Gamma_{0}\right)$ with integer $s>0$. From elliptic regularity theory it follows the inequality

$$
\|u\|_{s+t}^{\Gamma_{0}{ }^{2}} \leq C_{s, t} \sum_{|\alpha| \leq t}\left\|D^{\alpha} u\right\|_{s}^{\Gamma_{0}^{2}}
$$

for arbitrary $s, t \in \mathbb{N}$ and $u \in H^{s+t}\left(\Gamma_{0}\right)$.

We will use the notations $x_{n} \stackrel{x}{\longrightarrow} x$ for norm convergence and $x_{n} \stackrel{x}{\longrightarrow} x$ for weak convergence in the (Banach) space $X$.

Lemma 7 (Continuity of $\rho$ near 0 ). There is an $\varepsilon_{0}>0$ such that for all integers $s \geq s_{1}$ the mapping

$$
\rho: \quad B_{0}\left(\varepsilon_{0}, H^{s_{1}}\left(\Gamma_{0}\right)\right) \cap H^{s}\left(\Gamma_{0}\right) \longrightarrow H^{s-1}\left(\Gamma_{0}\right)
$$


(i) continuous and bounded

(ii) weakly sequentially continuous.

Proof. Statement (i): Set $s_{0}=s_{1}$. If $s=s_{1}$, then the assertion follows directly from Lemma 5 . If $s>s_{1}$, then because of (4.12) it is sufficient to show that the mappings $D^{\alpha} \rho$ are continuous and bounded from $B_{0}\left(\varepsilon_{0}, H^{s_{1}}\left(\Gamma_{0}\right)\right) \cap H^{s}\left(\Gamma_{0}\right)$ to $H^{s_{1}-1}\left(\Gamma_{0}\right)$ for all $\alpha$ with $|\alpha| \leq s-s_{1}$. Using (4.6) we find

$$
\begin{aligned}
&\left\|D^{\alpha}(\rho(r)-\rho(v))\right\|_{s_{1}-1}^{\Gamma_{0}} \\
& \leq \sum_{m=1}^{\infty}\left\|D^{\alpha} \rho_{m}(r, \ldots, r)-D^{\alpha} \rho_{m}(v, \ldots, v)\right\|_{s_{1}-1}^{\Gamma_{0}} \\
& \leq \sum_{l=0}^{m} \sum_{k=\max \{1, m-l)}^{|\alpha|} \sum_{\beta_{1}+\ldots+\beta_{k}=\alpha} C_{\beta_{1}, \ldots, \beta_{k}} \frac{(k+l) !}{l !(m-l) !(k-m+l) !} \\
& \times \sum_{\sigma}\left(\sum_{j=1}^{1} \| \rho_{k+l}\left(r, \ldots, r, r-v, v, \ldots, v, D^{\beta_{\sigma(1)}} v\right.\right. \\
&\left.\ldots, D^{\beta_{\sigma(m-l)}} v, D^{\beta_{\sigma(m-l+1)}} \mathcal{R}_{0}, \ldots, D^{\beta_{\sigma(k)}} \mathcal{R}_{0}\right) \|_{s_{1}-1}^{\Gamma_{0}} \\
&+\sum_{j=1}^{m-l} \| \rho_{k+l}\left(r, \ldots, r, D^{\beta_{\sigma(1)}} r, D^{\beta_{\sigma(j-1)} r, D^{\beta_{\sigma(j)}}(r-v), D^{\beta_{\sigma(j+l)}} v}\right. \\
&\left.\left.\quad \ldots, D^{\beta_{\sigma(m-l)}} v, D^{\beta_{\sigma(m-l+1)}} \mathcal{R}_{0}, \ldots, D^{\beta_{\sigma(k)}} \mathcal{R}_{0}\right) \|_{s_{1}-1}^{\Gamma_{0}}\right)
\end{aligned}
$$

with $r, v \in B_{0}\left(\varepsilon_{0}, H^{s_{1}}\left(\Gamma_{0}\right)\right) \cap H^{s}\left(\Gamma_{0}\right)$ and the understanding that in the first sum over $j$ the difference occurs in the $j$-th argument. We will estimate the summands of the sums in braces separately for $m>1$, using (3.9), the interpolation inequality

$$
\left\|D^{\beta} u\right\|_{s_{1}}^{\Gamma_{0}} \leq C\|u\|_{s_{1}+|\beta|}^{\Gamma_{0}} \leq C\|u\|_{s_{1}}^{\Gamma_{0}^{1}-\frac{\beta}{\mid a} \mid}\|u\|_{s}^{\Gamma_{0}} \frac{|\rho|}{a \mid}
$$

holding for all multiindices $\beta$ with $|\beta| \leq|\alpha|$ and the notations

$$
\left.\begin{array}{rl}
\mu_{s} & =\max \left\{\|u\|_{s}^{\Gamma_{0}},\|v\|_{s}^{\Gamma_{0}}\right\} \\
b & =\frac{\sum_{r=1}^{m-1}\left|\beta_{\sigma(r)}\right|}{|\alpha|} \\
\nu & =\frac{\left|\beta_{\sigma(j)}\right|}{|\alpha|} .
\end{array}\right\}
$$


We find for the summands in the first sum

$$
\begin{aligned}
& \| \rho_{k+l}\left(r, \ldots, r, r-v, v, \ldots, v, D^{\beta_{\sigma(1)}} v, \ldots\right. \\
&\left.D^{\beta_{\sigma(m-l)}} v, D^{\beta_{\sigma(m-l+1)}} \mathcal{R}_{0}, \ldots, D^{\beta_{\sigma(k)}} \mathcal{R}_{0}\right) \|_{s_{1}-1}^{\Gamma_{0}} \\
& \leq C_{s} M^{k+l} \mu_{s_{1}}^{l-1}\|v\|_{s_{1}}^{\Gamma_{0} m-1-b}\|v\|_{s}^{\Gamma_{0}^{b}}\|r-v\|_{s_{1}}^{\Gamma_{0}} \\
& \leq C_{s} M^{m} \mu_{s_{1}}^{m-1-b} \mu_{s}^{b}\|r-v\|_{s_{1}}^{\Gamma_{0}}
\end{aligned}
$$

and in the second sum

$$
\begin{aligned}
& \| \rho_{k+1}\left(r, \ldots, r, D^{\beta_{\sigma(1)}} r, D^{\beta_{\sigma(j-1)}} r, D^{\beta_{\sigma(j)}}(r-v), D^{\beta_{\sigma(j+1)}} v, \ldots\right. \\
&\left.D^{\beta_{\sigma(m-l)}} v, D^{\beta_{\sigma(m-l+1)}} \mathcal{R}_{0}, \ldots, D^{\beta_{\sigma(k)}} \mathcal{R}_{0}\right) \|_{s_{1}-1}^{\Gamma_{0}} \\
& \leq C_{s} M^{k+l}\|r\|_{s_{1}}^{\Gamma_{0}^{l}} \mu_{s_{1}}^{m-l-1-(b-\nu)} \mu_{s}^{b-\nu}\|r-v\|_{s}^{\Gamma_{0}} \\
& \leq C_{s} M^{m} \mu_{s_{1}}^{m-1-(b-\nu)} \mu_{s}^{b-\nu}\|r-v\|_{s}^{\Gamma_{0}} .
\end{aligned}
$$

Carrying out the summations over $\sigma, l, k$, and the $\beta$, we have to take into account that because of $l \leq m$ and $k \leq|\alpha|$

$$
\frac{(k+l) !}{l !} \leq(k+l)^{k} \leq(|\alpha|+m)^{|\alpha|} \leq 2^{|\alpha|}\left(|\alpha|^{|\alpha|}+m^{|\alpha|}\right)
$$

and this yields for small $\varepsilon_{0}$

$$
\begin{aligned}
\| D^{\alpha} \rho_{m}(r, \ldots, r) & -D^{\alpha} \rho_{m}(v, \ldots, v) \|_{s_{1}-1}^{\Gamma_{0}} \\
& \leq C_{9} M^{m}\left(1+m^{s-s_{1}}\right) \mu_{s_{1}-2}^{m-2}\left(1+\mu_{s}\right)\|r-v\|_{s}^{\Gamma_{0}} .
\end{aligned}
$$

Demanding now $\varepsilon_{0}<\frac{1}{M}$, using

$$
\left\|D^{\alpha} \rho_{1}(r-v)\right\|_{s_{1}-1}^{\Gamma_{0}} \leq C_{s}\|r-v\|_{s}^{\Gamma_{0}}
$$

and carrying out the summation over $m \geq 2$ yields

$$
\left\|D^{\alpha}(\rho(r)-\rho(v))\right\|_{s_{1}-1}^{\Gamma_{0}} \leq C_{s}\left(1+\mu_{s}\right)\|r-v\|_{s}^{\Gamma_{0}},
$$

and this estimate implies the boundedness and continuity of $D^{\alpha} \rho$.

(ii) From (i) and Lemma 5 with $s_{0}<s_{1}$ it follows that for any integer $s \geq s_{1}$ there is a $\tilde{s}<s$ such that the mapping

$$
\rho: B_{0}\left(\varepsilon_{0}, H^{s_{1}}\left(\Gamma_{0}\right)\right) \cap H^{s}\left(\Gamma_{0}\right) \longrightarrow H^{s-1}\left(\Gamma_{0}\right)
$$

is bounded and the mapping

$$
\rho: B_{0}\left(\varepsilon_{0}, H^{s_{1}}\left(\Gamma_{0}\right)\right) \cap H^{\bar{s}}\left(\Gamma_{0}\right) \longrightarrow H^{\bar{s}-1}\left(\Gamma_{0}\right)
$$


is continuous. For an arbitrary sequence $\left\{r_{n}\right\}, r_{n} \in B_{0}\left(\varepsilon_{0}, H^{s_{1}}\left(\Gamma_{0}\right)\right) \cap H^{9}\left(\Gamma_{0}\right)$ with $r_{n} \stackrel{H^{*}\left(\Gamma_{0}\right)}{\longrightarrow} r^{*}$ we have $r_{n} \stackrel{H^{i}\left(\Gamma_{0}\right)}{\longrightarrow} r^{*}$ and hence

$$
\rho\left(r_{n}\right) \stackrel{H^{j-1}\left(\Gamma_{0}\right)}{\longrightarrow} \rho\left(r^{*}\right)
$$

On the other hand, $\left\{\rho\left(r_{n}\right)\right\}$ is a bounded sequence in $H^{s-1}\left(\Gamma_{0}\right)$ and therefore it has a weakly convergent subsequence. Consider now an arbitrary weakly convergent subsequence $\left\{\rho\left(r_{n^{\prime}}\right)\right\}$ with $\rho\left(r_{n^{\prime}}\right) \stackrel{H^{*-1}\left(\Gamma_{0}\right)}{\longrightarrow} \rho^{*}$. This implies $\rho\left(r_{n^{\prime}}\right) \stackrel{H^{j-1}\left(\Gamma_{0}\right)}{\longrightarrow} \rho^{*}$ and thus, because of (4.15), $\rho^{*}=\rho\left(r^{*}\right)$. Hence we can conclude (see [22: Satz 10.2]) that $\rho\left(r_{n}\right) \stackrel{H^{*-1}\left(\Gamma_{0}\right)}{\longrightarrow} \rho\left(r^{*}\right)$

\section{Linearization}

For the further investigation of (3.7) one has to identify the operator $\rho_{1}$ more precisely. We find, using the notation of Section 3

$$
\begin{aligned}
\rho_{1}(r) & =\left(-\frac{u_{0} \cdot n}{\gamma^{2}} \zeta+\frac{1}{\gamma} u_{0}\right) \cdot \nu_{1}(r)+\frac{1}{\gamma} n \cdot u_{1}(r) \\
u_{1} & =\Pi_{1} L(0)^{-1}\left(F_{1}(r)-L_{1}(r) L(0)^{-1} F_{0}\right) .
\end{aligned}
$$

Calculating $F_{1}$ explicitly and recalling from Lemma $4 /(\mathrm{ii})$ that $\left\|L_{1}(r)\right\|_{\mathcal{L}(X, Y)} \leq C\|r\|_{\frac{1}{2}}^{\Gamma_{0}}$ we find that

$$
\rho_{1}(r)=\rho_{1}^{*}(r)+\Lambda_{1}(r)+\Lambda_{0}(r)
$$

with

$$
\rho_{1}^{\star}(r)=\frac{1}{\gamma}\left(\operatorname{Tr}_{\Gamma_{0}} \dot{u}\right) \cdot n
$$

and $[\dot{u} \dot{p} \dot{\lambda}]^{T} \in X$ the solution of the variational problem

$$
\begin{aligned}
a(\dot{u}, v)-\int_{\Omega_{0}} \dot{p} \operatorname{div} v d x+\dot{\lambda}_{1}^{T} \varphi_{1}(v)+\dot{\lambda}_{2}^{T} \varphi_{2}(v) & =\int_{\Gamma_{0}} \gamma \delta_{\Gamma_{0}} r n \cdot v d \Gamma_{0} \\
& \text { for all } v \in\left(H^{1}(\Omega)\right)^{N} \\
\operatorname{div} \dot{u} & =0 \\
\varphi_{1}(\dot{u}) & =0 \\
\varphi_{2}(\dot{u}) & =0
\end{aligned}
$$

with

$$
\Lambda_{1}(r)=\left(-\frac{u_{0} \cdot n}{\gamma^{2}} \zeta+\frac{1}{\gamma} u_{0}\right) \cdot \nu_{1}(r)
$$

a first order differential operator and

$$
\Lambda_{0} \in \mathcal{L}\left(H^{\frac{1}{2}}\left(\Gamma_{0}\right), H^{\frac{1}{2}}\left(\Gamma_{0}\right)\right) .
$$

By interpolation we have $\rho_{1} \in \mathcal{L}\left(H^{s}\left(\Gamma_{0}\right), H^{s-1}\left(\Gamma_{0}\right)\right)$ for all (real) $s \geq \frac{3}{2}$. 
Lemma 8 (Coercivity of $-\rho_{1}$ ). For all positive integer $s$ there are positive constants $c$, and $C$, such that

$$
-\left(\rho_{1} r, r\right)_{s} \geq c_{s}\|r\|_{s+\frac{1}{2}}^{\Gamma_{0}}{ }^{2}-C_{s}\|r\|_{s-\frac{1}{2}}^{\Gamma_{0}}{ }^{2} \quad \forall r \in H^{s+1}\left(\Gamma_{0}\right) .
$$

Proof. Step 1: $s=1$. We have

$$
\begin{aligned}
-\left(\rho_{1} r, r\right)_{H^{1}\left(\Gamma_{0}\right)} & \\
= & -\left(\int_{\Gamma_{0}} \nabla_{\Gamma_{0}} \rho_{1} r \cdot \nabla_{\Gamma_{0}} r d \Gamma_{0}+\int_{\Gamma_{0}} \rho_{1} r r d \Gamma_{0}\right) \\
\geq & -\left(\int_{\Gamma_{0}} \nabla_{\Gamma_{0}}\left(\rho_{1}^{\star} r+\Lambda_{1} r+\Lambda_{0} r\right) \cdot \nabla_{\Gamma_{0} r}\right)-C\|r\|_{\frac{3}{2}}^{\Gamma_{0}}\|r\|_{-\frac{1}{2}}^{\Gamma_{0}} \\
\geq & -\left(\int_{\Gamma_{0}} \nabla_{\Gamma_{0}} \rho_{1}^{\star} r \cdot \nabla_{\Gamma_{0}} r d \Gamma_{0}+\int_{\Gamma_{0}} \nabla_{\Gamma_{0}} \Lambda_{1} r \cdot \nabla_{\Gamma_{0}} r d \Gamma_{0}\right)-C\|r\|_{\frac{3}{2}}^{\Gamma_{0}}\|r\|_{\frac{1}{2}}^{\Gamma_{0}} .
\end{aligned}
$$

The two remaining integrals will be treated separately.

Substep 1.1. Problem (5.2) is the weak formulation of the problem

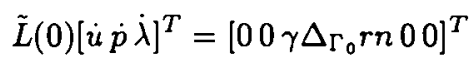

and from Lemma $2 /($ ii) we get

$$
\left\|\dot{\lambda}_{1}\right\|_{\mathbb{R}^{N}}+\left\|\dot{\lambda}_{2}\right\|_{\left.\left.\mathbb{R}^{(}\right)_{2}^{N}\right)} \leq C\left\|\gamma \Delta_{\Gamma_{0}} r n\right\|_{-\frac{3}{2}}^{\Gamma_{0}} \leq C\|r\|_{\frac{1}{2}}^{\Gamma_{0}} .
$$

In order to give an estimate for $\dot{p}$, consider the following Neumann problem for the Laplacian:

$$
\left.\begin{array}{ll}
\Delta \Phi=\dot{p} & \text { in } \Omega_{0} \\
\frac{\partial \Phi}{\partial n}=\gamma^{-1} \frac{\int_{\Omega_{0}} \dot{p} d x}{\int_{\Gamma_{0}} \gamma^{-1} d \Gamma_{0}}=g & \text { on } \Gamma_{0} .
\end{array}\right\}
$$

It is solvable because

$$
\int_{\Gamma_{0}} g d \Gamma_{0}=\int_{\Omega_{0}} \dot{p} d x
$$

and because of $\dot{p} \in L^{2}\left(\Omega_{0}\right)$ and $g \in H^{\frac{1}{2}}\left(\Gamma_{0}\right)$ the regularity theory for this problem yields the existence of a solution $\Phi \in H^{2}\left(\Omega_{0}\right)$ satisfying the estimate

$$
\|\Phi\|_{2}^{\Omega_{0}} \leq C\left(\|\dot{p}\|_{0}^{\Omega_{0}}+\|g\|_{\frac{1}{2}}^{\Gamma_{0}}\right) \leq C\|\dot{p}\|_{0}^{\Omega_{0}} .
$$

If we set now $v=v_{p}=\nabla \Phi$ in the first equation of (5.2) and take into account that $\operatorname{div} v_{p}=\dot{p},\left\|v_{p}\right\|_{1}^{\Omega_{0}} \leq\|\Phi\|_{2}^{\Omega_{0}} \leq C\|\dot{p}\|_{0}^{\Omega_{0}}, \gamma n \cdot v_{p}$ is constant on $\Gamma_{0}$ and thus the boundary integral in (5.2) vanishes, we find

$$
\begin{aligned}
\|\dot{p}\|_{0}^{\Omega_{0}^{2}} & \leq\left|a\left(\dot{u}, v_{p}\right)\right|+\left|\dot{\lambda}_{1}^{T} \varphi_{1}\left(v_{p}\right)\right|+\left|\dot{\lambda}_{2}^{T} \varphi_{2}\left(v_{p}\right)\right| \\
& \leq C\left(\|\dot{u}\|_{1}^{\Omega_{0}}+\|r\|_{0}^{\Gamma_{0}}\right)\left\|v_{p}\right\|_{1}^{\Omega_{0}} \\
& \leq C\left(\|\dot{u}\|_{1}^{\Omega_{0}}+\|r\|_{0}^{\Gamma_{0}}\right)\|\dot{p}\|_{0}^{\Omega_{0}}
\end{aligned}
$$


and hence

$$
\|\dot{p}\|_{0}^{\Omega_{0}} \leq C\left(\|\dot{u}\|_{1}^{\Omega_{0}}+\|r\|_{0}^{\Gamma_{0}}\right) .
$$

The positive smooth function $\gamma$ has a positive smooth extension to $\Omega_{0}$ which will be denoted by the same symbol. So we get for the first integral by the Green formula from (5.2) and the ellipticity of $a$, using the generalized Schwarz inequality and the estimate

$$
\|\dot{u}\|_{0}^{\Omega_{0}} \leq C\left\|\gamma \Delta_{\Gamma_{0}} r n\right\|_{-\frac{3}{2}}^{\Gamma_{0}} \leq C\|r\|_{\frac{1}{2}}^{\Gamma_{0}}
$$

from Lemma 2/(ii)

$$
\begin{aligned}
&-\int_{\Gamma_{0}} \nabla_{\Gamma_{0}} \rho_{1}^{*} r \cdot \nabla_{\Gamma_{0}} r d \Gamma_{0} \\
&= \int_{\Gamma_{0}} \frac{\dot{u}}{\gamma^{2}} \cdot \gamma n \Delta_{\Gamma_{0}} r d \Gamma_{0} \\
&= a\left(\dot{u}, \frac{\dot{u}}{\gamma^{2}}\right) \\
&= \frac{1}{2} \sum_{i, j=1}^{N}\left(\int_{\Omega_{0}} \gamma^{-2}\left(\frac{\partial \dot{u}_{i}}{\partial x_{j}}+\frac{\partial \dot{u}_{j}}{\partial x_{i}}\right)^{2} d x\right. \\
&\left.+\int_{\Omega_{0}}\left(\frac{\partial \dot{u}_{i}}{\partial x_{j}}+\frac{\partial \dot{u}_{j}}{\partial x_{i}}\right)\left(\dot{u}_{i} \frac{\partial\left(\gamma^{-2}\right)}{\partial x_{j}}+\dot{u}_{j} \frac{\partial\left(\gamma^{-2}\right)}{\partial x_{i}}\right) d x\right) \\
& \geq c\|\dot{u}\|_{1}^{\Omega_{0}^{2}}-C\|\dot{u}\|_{1}^{\Omega_{0}}\|\dot{u}\|_{0}^{\Omega_{0}} \\
& \geq c\|\dot{u}\|_{1}^{\Omega_{0}^{2}}-C\|\dot{u}\|_{0}^{\Omega_{0}^{2}} \\
& \geq c\|\dot{u}\|_{1}^{\Omega_{0}^{2}}-C\|r\|_{\frac{1}{2}}^{\Gamma_{0}^{2}} .
\end{aligned}
$$

On the other hand,

$$
\begin{aligned}
\|r\|_{\frac{3}{2}}^{\Gamma_{0}} & \leq C\left\|\Delta_{\Gamma_{0}} r\right\|_{-\frac{1}{2}}^{\Gamma_{0}}+C\|r\|_{\frac{1}{2}}^{\Gamma_{0}} \\
& \leq C \sup \left\{\int_{\Gamma_{0}} \Delta_{\Gamma_{0}} r \varphi d \Gamma_{0} \mid \varphi \in H^{\frac{1}{2}}\left(\Gamma_{0}\right),\|\varphi\|_{\frac{1}{2}}^{\Gamma_{0}}=1\right\}+C\|r\|_{\frac{1}{2}}^{\Gamma_{0}} .
\end{aligned}
$$

For any $\varphi \in H^{\frac{1}{2}}\left(\Gamma_{0}\right)$ with $\|\varphi\|_{\frac{1}{2}}^{\Gamma_{0}^{0}}=1$ define now the constant

$$
\bar{\varphi}=\frac{\int_{\Gamma_{0}} \gamma^{-1} \varphi d \Gamma_{0}}{\int_{\Gamma_{0}} \gamma^{-1} d \Gamma_{0}}
$$

for which $\|\bar{\varphi}\|_{\frac{1}{2}}^{\Gamma_{0}} \leq C|\bar{\varphi}| \leq C\|\varphi\|_{0}^{\Gamma_{0}} \leq C$ holds. Consider again a Neumann problem

$$
\left.\begin{array}{ll}
\Delta \Phi=0 & \text { in } \Omega_{0} \\
\frac{\partial \Phi}{\partial n}=\gamma^{-1}(\varphi-\bar{\varphi}) & \text { at } \Gamma_{0} .
\end{array}\right\}
$$


Because of $\int_{\Gamma_{0}} \gamma^{-1}(\varphi-\bar{\varphi}) d \Gamma_{0}=0$ it has a solution $\Phi \in H^{2}\left(\Omega_{0}\right)$ with

$$
\|\Phi\|_{2}^{\Omega_{0}} \leq C\left\|\gamma^{-1}(\varphi-\bar{\varphi})\right\|_{\frac{1}{2}}^{\Gamma_{0}} \leq C\left(\|\varphi\|_{\frac{1}{2}}^{\Gamma_{0}}+\|\bar{\varphi}\|_{\frac{1}{2}}^{\Gamma_{0}}\right) \leq C .
$$

If we define again $v=\nabla \Phi$ we find $v \in\left(H^{1}\left(\Omega_{0}\right)\right)^{N},\|v\|_{1}^{\Omega_{0}} \leq C$, and with (5.2)

$$
\begin{aligned}
\int_{\Gamma_{0}} \Delta \Gamma_{0} r \varphi d \Gamma_{0} & =\int_{\Gamma_{0}} \Delta_{\Gamma_{0}} r(\varphi-\bar{\varphi}) d \Gamma_{0} \\
& =\int_{\Gamma_{0}} \gamma \Delta_{\Gamma_{0}} r n \cdot v d \Gamma_{0} \\
& =a(\dot{u}, v)-\int_{\Omega_{0}} \dot{p} \operatorname{div} v d x+\dot{\lambda}_{1}^{T} \varphi_{1}(v)+\dot{\lambda}_{2}^{T} \varphi_{2}(v) \\
& \leq C\|(\dot{u}, \dot{p}, \dot{\lambda})\|_{X} \\
& \leq C\left(\|\dot{u}\|_{1}^{\Omega_{0}}+\|r\|_{\frac{1}{2}}^{\Gamma_{0}}\right)
\end{aligned}
$$

where (5.3) and (5.4) have been used. Hence, together with (5.6),

$$
\|r\|_{\frac{3}{2}}^{\Gamma_{0}^{2}} \leq C\left(\|\dot{u}\|_{1}^{\Omega_{0}^{2}}+\|r\|_{\frac{1}{2}}^{\Gamma_{0}}\right)
$$

and with (5.5)

$$
-\int_{\Gamma_{0}} \nabla_{\Gamma_{0}} \rho_{1}^{\star} r \cdot \nabla_{\Gamma_{0}} r d \Gamma_{0} \geq c\|r\|_{\frac{3}{2}}^{\Gamma_{0}^{2}}-C\|r\|_{\frac{1}{2}}^{\Gamma_{0}^{2}} .
$$

Substep 1.2: Next, we have to deal with the integral

$$
\begin{aligned}
\int_{\Gamma_{0}} & \nabla_{\Gamma_{0}} \Lambda_{1} r \cdot \nabla_{\Gamma_{0}} r d \Gamma_{0} \\
& =-\int_{\Gamma_{0}} \Lambda_{1} r \Delta_{\Gamma_{0}} r d \Gamma_{0} \\
& =-\int_{\Gamma_{0}} r \Lambda_{1}^{*} \Delta_{\Gamma_{0}} r d \Gamma_{0} \\
& =\int_{\Gamma_{0}} r \Lambda_{1} \Delta_{\Gamma_{0}} r d \Gamma_{0}-\int_{\Gamma_{0}} r\left(\Lambda_{1}+\Lambda_{1}^{*}\right) \Delta_{\Gamma_{0}} r d \Gamma_{0} \\
& =\int_{\Gamma_{0}} r \Delta_{\Gamma_{0}} \Lambda_{1} r d \Gamma_{0}+\int_{\Gamma_{0}} r\left(\Lambda_{1} \Delta_{\Gamma_{0}}-\Delta_{\Gamma_{0}} \Lambda_{1}\right) r d \Gamma_{0}-\int_{\Gamma_{0}} r\left(\Lambda_{1}+\Lambda_{1}^{*}\right) \Delta_{\Gamma_{0}} r d \Gamma_{0} \\
& =\int_{\Gamma_{0}} \Delta_{\Gamma_{0}} r \Lambda_{1} r d \Gamma_{0}+\int_{\Gamma_{0}} r \Lambda_{2} r d \Gamma_{0}
\end{aligned}
$$

where $\Lambda_{1}^{*}$ denotes the adjoint of $\Lambda_{1}$ in $H^{0}\left(\Gamma_{0}\right)$ and

$$
\Lambda_{2}=\Lambda_{1} \Delta_{\Gamma_{0}}-\Delta_{\Gamma_{0}} \Lambda_{1}-\left(\Lambda_{1}+\Lambda_{1}^{*}\right) \Delta_{\Gamma_{0}}
$$


$\Lambda_{2}$ is a second order differential operator due to the well-known facts that the commutator $\Lambda_{1} \Delta_{\Gamma_{0}}-\Delta_{\Gamma_{0}} \Lambda_{1}$ is a differential operator of second order only and $\Lambda_{1}+\Lambda_{1}^{*}$ is given purely by multiplication with a smooth function. Hence

$$
\left|\int_{\Gamma_{0}} \nabla_{\Gamma_{0}} \Lambda_{1} r \cdot \nabla_{\Gamma_{0}} r d \Gamma_{0}\right|=\frac{1}{2}\left|\int_{\Gamma_{0}} r \Lambda_{2} r d \Gamma_{0}\right| \leq C\|r\|_{\frac{1}{2}}^{\Gamma_{0}}\left\|\Lambda_{2} r\right\|_{-\frac{1}{2}}^{\Gamma_{0}} \leq C\|r\|_{\frac{1}{2}}^{\Gamma_{0}}\|r\|_{\frac{3}{2}}^{\Gamma_{0}} .
$$

The. assertion for $s=1$ follows now by summing up and applying the generalized Schwarz inequality again.

Step 2: $1<s \leq s_{1}$. We have, using Step 1 of the proof, (4.7) and the generalized Schwarz inequality

$$
\begin{aligned}
-\left(\rho_{1} r, r\right)_{s}= & -\sum_{|\alpha| \leq s-1}\left(D^{\alpha} \rho_{1} r, D^{\alpha} r\right)_{1} \\
= & -\sum_{|\alpha| \leq s-1}\left(\left(\left(D^{\alpha} \rho_{1}-\rho_{1} D^{\alpha}\right) r, D^{\alpha} r\right)_{1}+\left(\rho_{1} D^{\alpha} r, D^{\alpha} r\right)_{1}\right) \\
\geq & c \sum_{|\alpha| \leq s-1}\left\|D^{\alpha} r\right\|_{\frac{3}{2}}^{\Gamma_{0}^{2}} \\
& -C \sum_{|\alpha| \leq s-1}\left(\left\|\left(D^{\alpha} \rho_{1}-\rho_{1} D^{\alpha}\right) r\right\|_{\frac{1}{2}}^{\Gamma_{0}}\left\|D^{\alpha} r\right\|_{\frac{3}{2}}^{\Gamma_{0}}+\left\|D^{\alpha} r\right\|_{\frac{1}{2}}^{\Gamma_{0}^{2}}\right) \\
\geq & c_{s}\|r\|_{s+\frac{1}{2}}^{\Gamma_{0}} 2
\end{aligned}
$$

Step 3: $s>s_{1}$. The proof can be given as in Step 2, using (4.8) instead of (4.7)

\section{Existence and uniqueness for the nonlinear problem}

Due to its analyticity, the behavior of the operator $\rho$ is locally governed by its linearization $\rho_{1}$. This and the chain rule enable us to show the following estimate.

Lemma 9 (Local a priori estimate). There is an $\varepsilon_{1}>0$ such that for all integer $s>s_{1}$ an inequality

$$
(\rho(r), r)_{s} \leq-c_{s}\|r\|_{s+\frac{1}{2}}^{\Gamma_{0}}{ }^{2}+C_{s}\left(\|r\|_{s-\frac{1}{2}}^{\Gamma_{0}}{ }^{2}+1\right)
$$

holds for all $r \in B_{0}\left(\varepsilon_{1}, H^{s_{1}+1}\left(\Gamma_{0}\right)\right) \cap H^{s+1}\left(\Gamma_{0}\right)$.

Proof. We demand $\varepsilon_{1} \leq \varepsilon_{0}$ and conclude from Lemma $7 /(\mathrm{i})$ that $\rho(r) \in H^{s}\left(\Gamma_{0}\right)$. We decompose

$$
\rho(r)=\rho(0)+\rho_{1} r+\sum_{m=2}^{\infty} \rho_{m}(r, \ldots, r)
$$

and use (4.10). For the sake of brevity we will restrict our attention to the case $|\alpha|>0$, the estimates for $\alpha=0$ are obvious. 
1. Because $\rho(0)$ is smooth we have

$$
\left(D^{\alpha} \rho(0), D^{\alpha} r\right)_{s_{1}} \leq C_{s}\|r\|_{g-\frac{1}{2}}^{\Gamma_{0}} \leq C_{s}\left(\|r\|_{g-\frac{1}{2}}^{\Gamma_{0}}{ }^{2}+1\right) .
$$

2. From the proof of Lemma 8 we recall

$$
\left(D^{\alpha} \rho_{1} r, D^{\alpha} r\right)_{s_{1}} \leq-c^{*}\left\|D^{\alpha} r\right\|_{s_{1}-\frac{1}{2}}^{\Gamma_{0}}{ }^{2}+C,\|r\|_{s-\frac{1}{2}}^{\Gamma_{0}}{ }^{2} .
$$

3. We use (4.6) and estimate

$$
\begin{aligned}
&\left(D^{\alpha} \rho_{m}(r, \ldots, r), D^{\alpha} r\right)_{s_{1}} \\
& \leq C\left\|D^{\alpha} \rho_{m}(r, \ldots, r)\right\|_{g_{1}-\frac{1}{2}}^{\Gamma_{0}}\left\|D^{\alpha} r\right\|_{s_{1}+\frac{1}{2}}^{\Gamma_{0}} \\
& \leq C \sum_{l=0}^{m} \sum_{k=\max \{1, m-l\}}^{|\alpha|} \sum_{\beta_{1}+\ldots+\beta_{k}=\alpha} C_{\beta_{1}, \ldots, \beta_{k}} \frac{(k+l) !}{l !(m-l) !(k-m+l) !} \\
& \times \sum_{\sigma} T_{k+l, m, \underline{\beta}, \sigma}\left\|D^{\alpha} r\right\|_{s_{1}+\frac{1}{2}}^{\Gamma_{0}}
\end{aligned}
$$

with the shorthand notations

$$
\begin{aligned}
\underline{\beta} & =\left(\beta_{1}, \ldots, \beta_{k}\right) \\
T_{k+l, m, \underline{\beta}, \sigma} & =\left\|\rho_{k+l}\left(r, \ldots, r, D^{\beta_{\sigma(1)}} r, \ldots, D^{\beta_{\sigma(m-1)}} r, D^{\beta_{\sigma(m-1+1)}} \mathcal{R}_{0}, D^{\beta_{\sigma(k)}} \mathcal{R}_{0}\right)\right\|_{g_{1}-\frac{1}{2}}^{\Gamma_{0}}
\end{aligned}
$$

which will be continuously used in the sequel. We will estimate the terms $T_{k+l, m, \beta}, \sigma$ separately and then perform the summations.

Note at first that the sum over $\sigma$ has $k$ ! elements and that due to $k \leq|\alpha|$ and $l \leq m$ we have

$$
\frac{(k+l) ! k !}{l !(m-l) !(k-m+l) !}=\frac{(k+l) !}{l !}\left(\begin{array}{c}
k \\
m-l
\end{array}\right) \leq(m+|\alpha|)^{|\alpha|} 2^{|\alpha|-1} \leq C_{\alpha} m^{|\alpha|}
$$

Take now $m \geq 2, l, k, \beta$, and $\sigma$ fixed. We will distinguish several cases and continuously use the estimates for the $\rho_{k}$ together with (3.9).

Case 3.1: $k+l=m$.

Subcase 3.1.1: $k=1$. To this choice of the indices there corresponds only the term

$$
m T_{m, m,(\alpha),(1)} \leq C m M^{m}\|r\|_{s_{1}+\frac{1}{2}}^{\Gamma_{0}}{ }^{m-1}\left\|D^{\alpha} r\right\|_{s_{1}+\frac{1}{2}}^{\Gamma_{0}} .
$$

If we perform the summation over $m \geq 2$ and choose $\varepsilon_{1}$ small enough we get

$$
\sum_{m=2}^{\infty} m T_{m, m,(\alpha),(1)}\left\|D^{\alpha} r\right\|_{s_{1}+\frac{1}{2}}^{\Gamma_{0}} \leq \frac{c^{*}}{4}\left\|D^{\alpha} r\right\|_{s_{1}+\frac{1}{2}}^{\Gamma_{0}}{ }^{2}
$$


Subcase 3.1.2: $k>1$. In this case we have $\left|\beta_{j}\right|<|\alpha|$ for all $j$ and using the interpolation inequalities

$$
\begin{aligned}
\left\|D^{\beta} r\right\|_{s_{1}+\frac{1}{2}}^{\Gamma_{0}} & \leq C_{s}\|r\|_{s_{1}+|\beta|+\frac{1}{2}}^{\Gamma_{0}} \leq C_{s}\|r\|_{g_{1}+1}^{\Gamma_{0} 1-|\beta|}\|r\|_{s+\frac{1}{2}-\zeta}^{\Gamma_{0}} \frac{|\beta|}{|a|} \\
\|r\|_{s+\frac{1}{2}-\zeta}^{\Gamma_{0}} & \leq \delta\|r\|_{s+\frac{1}{2}}^{\Gamma_{0}}+C_{s, \delta}\|r\|_{s_{1}+1}^{\Gamma_{0}}
\end{aligned}
$$

holding for sufficiently small $\zeta$ depending only on $s$, all positive $\delta$ and all $\beta$ with $|\beta|<|\alpha|$ we find

$$
T_{m, m, \underline{\beta}, \sigma} \leq C, M^{m} m^{s-s_{1}}\|r\|_{s_{1}+1}^{\Gamma_{0}}{ }^{m-1}\left(\delta\|r\|_{s+\frac{1}{2}}^{\Gamma_{0}}+C, \delta\|r\|_{s_{1}+1}^{\Gamma_{0}}\right) \text {. }
$$

The convergence radius of the series $\sum_{m=2}^{\infty} M^{m} m^{|\alpha|} \varepsilon^{m-1}$ is $\frac{1}{M}$ and thus independent of $s$. Hence, performing the summations over $k, \beta, \sigma$, and $m$, using (6.1), applying the generalized Schwarz inequality and choosing $\delta$ sufficiently small we find

$$
\sum_{k>1, \underline{\beta}, \sigma, m} C_{\underline{\beta}} T_{m, m, \underline{\beta}, \sigma}\left\|D^{\alpha} r\right\|_{s_{1}+\frac{1}{2}}^{\Gamma_{0}} \leq \frac{c_{s} c^{*}}{4 \mathcal{N}_{s}}\|r\|_{s+\frac{1}{2}}^{\Gamma_{0}}{ }^{2}+C_{s}
$$

where $\mathcal{N}_{s}$ is the number of elements of the set $\{\alpha:|\alpha| \leq s\}$ and $c_{s}$ is a small positive constant such that

$$
\sum_{|\alpha| \leq s-s_{1}}\left\|D^{\alpha} r\right\|_{s_{1}+\frac{1}{2}}^{\Gamma_{0}}{ }^{2} \geq c_{s}\|r\|_{s+\frac{1}{2}}^{\Gamma_{0} 2}
$$

Case 3.2: $k+l>m$. In this case

$$
b=\frac{1}{|\alpha|} \sum_{j=1}^{m-1}\left|\beta_{\sigma(j)}\right|<1
$$

and by interpolation and Youngs inequality

$$
\begin{aligned}
T_{k+l, m, \underline{\beta}, \sigma} & \leq C_{s} M^{m}\|r\|_{s_{1}+1}^{\Gamma_{0}}{ }^{m-1}\|r\|_{s_{1}+1}^{\Gamma_{0}{ }^{1-b}\|r\|_{g+\frac{1}{2}}^{\Gamma_{0} b}} \\
& \leq C_{s} M^{m}\|r\|_{s_{1}+1}^{\Gamma_{0}{ }^{m-1}}\left(\delta\|r\|_{s+\frac{1}{2}}^{\Gamma_{0}}+C_{s, \delta}\|r\|_{s_{1}+1}^{\Gamma_{0}}\right)
\end{aligned}
$$

for any $\delta>0$. In the same way as in the previous case we find from this

$$
\sum_{m=2}^{\infty} \sum_{k, l, \underline{\beta}, \sigma} T_{k+l, m, \underline{\beta}, \sigma}\left\|D^{\alpha} r\right\|_{s_{1}+\frac{1}{2}}^{\Gamma_{0}} \leq \frac{c_{s} c^{*}}{4 \mathcal{N}_{s}}\|r\|_{s+\frac{1}{2}}^{\Gamma_{0}}{ }^{2}+C_{s}
$$

and the lemma is proved by carrying out the remaining summations

Now we can prove a short-time existence result for the solution of our evolution problem. As in [15] we will use the notations $I T$ for the closed interval $[0, T](T>0)$, $C_{w}(I T, X)$ and $C_{w}^{k}(I T, X)$ for the spaces of weakly continuous and $k$ times weakly differentiable functions valued in some Banach space $X$, respectively, i.e. the functions $u: I T \longrightarrow X$ such that, for all $\varphi \in X^{\prime},\langle\varphi, u(t)\rangle \in C(I T)$ and $\langle\varphi, u(t)\rangle \in C^{k}(I T)$, respectively. 
Proposition 1 (Short-time existence). Let $\Omega_{0}$ be smooth and strictly star-shaped. There are positive constants $\varepsilon_{2}$ and $T$ such that for all integer $s \geq s_{1}$ and all

$$
r_{0} \in B_{0}\left(\varepsilon_{2}, H^{s_{1}+1}\left(\Gamma_{0}\right)\right) \cap H^{s+1}\left(\Gamma_{0}\right)
$$

the initial value problem

$$
\left.\begin{array}{rl}
\frac{\partial r}{\partial t} & =\rho(r) \\
r(0) & =r_{0}
\end{array}\right\}
$$

has a solution $r$ in $C_{w}\left(I T, H^{s+1}\left(\Gamma_{0}\right)\right) \cap C_{w}^{1}\left(I T, H^{s}\left(\Gamma_{0}\right)\right)$.

Proof. The proof will be given in essentially the same way as the proof of Theorem A in [15] where $H^{s+2}\left(\Gamma_{0}\right), H^{s+1}\left(\Gamma_{0}\right)$, and $H^{s}\left(\Gamma_{0}\right)$ will play the roles of $V, H$, and $X$, respectively. The necessary modifications are due to the fact that both the estimate

$$
(\rho(r), r)_{s+1} \leq C_{s}\left(1+\|r\|_{s+1}^{r_{0}}{ }^{2}\right)
$$

and the weak continuity of $\rho$ are ensured by the Lemmas 9 and $7 /(\mathrm{ii})$ only if $\|r\|_{s_{1}+1}^{\Gamma_{0}} \leq$ $2 \varepsilon_{2}$ with sufficiently small $\varepsilon_{2}$. Thus we have to use Galerkin approximations which remain small in $H^{s_{1}+1}\left(\Gamma_{0}\right)$ and uniformly bounded in $H^{s+1}\left(\Gamma_{0}\right)$.

If $s>s_{1}$, then there is a self-adjoint operator $S$ on $H^{s_{1}+1}\left(\Gamma_{0}\right)$ such that

$$
(u, v)_{s_{+1}}=(S u, v)_{s_{1}+1} \quad \forall u \in D(S), v \in H^{s+1}\left(\Gamma_{0}\right) \text {. }
$$

By Rellich's theorem, $S$ has a purely discrete spectrum, i.e. $S$ has a complete orthonormal system of eigenfunctions $\left\{e_{j}\right\}$ in $H^{s_{1}+1}\left(\Gamma_{0}\right)$. Elliptic regularity theory yields that all $e_{j}$ are smooth. If $s=s_{1}$, then we choose an arbitrary orthonormal basis $\left\{e_{j}\right\}$ in $H^{s_{1}+1}\left(\Gamma_{0}\right)=H^{s+1}\left(\Gamma_{0}\right)$ consisting of functions in $H^{s+2}\left(\Gamma_{0}\right)$. We define now

$$
\begin{aligned}
& M_{k}=\operatorname{span}\left\{e_{1}, \ldots, e_{k}\right\} \\
& P_{k} u=\sum_{j=1}^{k}\left(u, e_{j}\right)_{s_{1}+1} e_{j}
\end{aligned}
$$

and it is easily seen that $P_{k}$ is the orthogonal projection on $M_{k}$ both in $H^{s_{1}+1}\left(\Gamma_{0}\right)$ and $H^{s+1}\left(\Gamma_{0}\right)$.

Consider the unique solution $m$ of the initial value problem

$$
\left.\begin{array}{rl}
\dot{m} & =2 C_{s_{1}}(1+m) \\
m(0) & =\varepsilon_{2}^{2}
\end{array}\right\}
$$

where $C_{s_{1}}$ is the constant $C_{s}$ from (6.3) with $s=s_{1}$ and choose $T$ to be the (uniquely defined) positive number satisfying $m(T)=4 \varepsilon_{2}^{2}$. Note that $m$ is strictly increasing on $I T$. We will show now that the Galerkin approximations $r$; defined by

$$
\left.\begin{array}{rl}
\frac{\partial r_{j}}{\partial t} & =P_{j} \rho\left(r_{j}\right) \\
r_{j}(0) & =P_{j} r_{0}
\end{array}\right\}
$$


exist at least at $I T$ and satisfy

$$
\left\|r_{j}(t)\right\|_{s_{1}+1}^{\Gamma_{0}}<2 \varepsilon_{2} \quad \forall j \in \mathbb{N}, t \in I T .
$$

Suppose the opposite: this implies by the theory of ordinary differential equations that for a certain $j$ there is a $T^{*}<T$ such that $\left\|r_{j}\left(T^{*}\right)\right\|_{9_{1}+1}^{\Gamma_{0}}=2 \varepsilon_{2}$ and $\left\|r_{j}(t)\right\|_{9_{1}+1}^{\Gamma_{0}}<2 \varepsilon_{2}$ for all $t \in\left[0, T^{*}\right)$. Note that $T^{*}>0$ because of

$$
\left\|r_{j}(0)\right\|_{s_{1}+1}^{\Gamma_{0}}=\left\|P_{j} r_{0}\right\|_{s_{1}+1}^{\Gamma_{0}} \leq \varepsilon_{2}
$$

For all $t \in I T^{*}$ we can estimate, by (6.3) and the same arguments as in [15],

$$
\frac{d}{d t}\left(\left\|r_{j}(t)\right\|_{s_{1}+1}^{\Gamma_{0}-2}\right) \leq 2 C_{s_{1}}\left(1+\left\|r_{j}(t)\right\|_{s_{1}+1}^{\Gamma_{0}}{ }^{2}\right)
$$

and from this and (6.6) an elementary comparison result for the solutions of initial value problems of ordinary differential equations in $\mathbb{R}$ yields

$$
m(T)=4 \varepsilon_{2}^{2}=\left\|r_{j}\left(T^{*}\right)\right\|_{s_{1}+1}^{\Gamma_{0}}{ }^{2} \leq m\left(T^{*}\right)
$$

in contradiction to the strict increasing of $m$. Hence (6.5) holds, and therefore, by repeating the above arguments for the $H^{s+1}\left(\Gamma_{0}\right)$-norm,

$$
\begin{aligned}
\frac{d}{d t}\left(\left\|r_{j}(t)\right\|_{s+1}^{\Gamma_{0}}{ }^{2}\right) & \leq 2 C_{s}\left(1+\left\|r_{j}(t)\right\|_{s+1}^{\Gamma_{0}}{ }^{2}\right) \\
\left\|r_{j}(0)\right\|_{s+1}^{\Gamma_{0}{ }^{2}} & \leq\left\|r_{0}\right\|_{s+1}^{\Gamma_{0}{ }^{2}}
\end{aligned}
$$

which implies that $\left\|r_{j}(t)\right\|_{s+1}^{\Gamma_{0}}$ exists and is bounded independently of $j$ on $I T$. The existence proof can be given now in strict analogy to the proof in [15] mentioned above

Taking into account that $C_{w}^{1}\left(I T, H^{s}\left(\Gamma_{0}\right)\right) \subset C^{1}\left(I T, H^{s-1}\left(\Gamma_{0}\right)\right)$ and the embedding theorems we immediately find:

Corollary 1. Under the assumptions of Proposition 1, suppose additionally $r_{0} \in$ $C^{\infty}\left(\Gamma_{0}\right)$. Then (6.2) has a solution in $C^{1}\left(I T, C^{\infty}\left(\Gamma_{0}\right)\right)$.

Lemma 10 (Weakened local monotonicity). For all $s \geq s_{1}$ there are positive constants $c_{s}, C_{s}$, and $\varepsilon_{s}$ such that

$$
\begin{aligned}
(\rho(r)- & \rho(v), r-v)_{s+1} \\
\leq & -c_{s}\|r-v\|_{s+\frac{3}{2}}^{\Gamma_{0}}{ }^{2}+C_{s}\|r-v\|_{s+\frac{1}{2}}^{\Gamma_{0}}{ }^{2} \\
& \quad+C_{s} \max \left\{\|r\|_{s+\frac{3}{2}}^{\Gamma_{0}},\|v\|_{s+\frac{3}{2}}^{\Gamma_{0}}\right\}\|r-v\|_{s+\frac{3}{2}}^{\Gamma_{0}}\|r-v\|_{s+1}^{\Gamma_{0}}
\end{aligned}
$$

for all $r, v \in B_{0}\left(\varepsilon_{s}, H^{s+1}\left(\Gamma_{0}\right)\right) \cap H^{s+2}\left(\Gamma_{0}\right)$. 
Proof. We proceed similar to the proof of Lemma 9 and use the notation (4.13) again. We find

$$
\begin{aligned}
& (\rho(r)-\rho(v), r-v)_{s+1} \\
& \leq-c^{*}\|r-v\|_{s+\frac{3}{2}}^{\Gamma_{0}}{ }^{2}+C\|r-v\|_{s+\frac{1}{2}}^{\Gamma_{0}}{ }^{2}+C \sum\left(1+m^{3}\right) \\
& \times\left(\sum_{j=1}^{l} \| \rho_{k+1}\left(r, \ldots, r, r-v, v, \ldots, v, D^{\beta_{\sigma(1)}} v, \ldots, D^{\beta_{\bullet(m-1)}} v,\right.\right. \\
& \left.D^{\beta_{\sigma(m-1+1)}} \mathcal{R}_{0}, \ldots, D^{\beta_{\bullet(k)}} \mathcal{R}_{0}\right) \|_{\rho_{1}-\frac{1}{2}}^{\Gamma_{0}} \\
& +\sum_{j=1}^{m} \| \rho_{k+l}\left(r, \ldots, r, D^{\beta_{\sigma(1)}} r, \ldots, D^{\beta_{\sigma(j-1)}} r, D^{\beta_{\sigma(j)}}(r-v), D^{\beta_{\sigma(j+1)}} v, \ldots,\right. \\
& \left.\left.D^{\beta_{\sigma(m-1)}} v, D^{\beta_{\sigma(m-1+1)}} \mathcal{R}_{0}, \ldots, D^{\beta_{\sigma(k)}} \mathcal{R}_{0}\right) \|_{g_{1}-\frac{1}{2}}^{\Gamma_{0}}\right)\|r-v\|_{s+\frac{3}{2}}^{\Gamma_{0}}
\end{aligned}
$$

where the sum has to be taken over $\alpha, k, \beta_{1}, \ldots, \beta_{k}, l, m$, and $\sigma$ as in Lemma 7 . The summands in brackets can be estimated by

or

$$
C M^{m}\left(1+m^{s}\right) \mu_{s+1}^{m-2} \mu_{s+\frac{3}{2}}\|r-v\|_{s+1}^{\Gamma_{0}}\|r-v\|_{s+\frac{3}{2}}^{\Gamma_{0}}
$$

$$
C M^{m}\left(1+m^{s}\right) \mu_{s+1}^{m-1}\|r-v\|_{s+\frac{3}{2}}^{\Gamma_{0}}{ }^{2}
$$

depending on whether the derivatives of highest order occur in the argument containing $r-v$. Choosing $\varepsilon$, small enough and carrying out the summations completes the proof

Proposition 2 (Uniqueness). Let $\Omega_{0}$ be as in Proposition 1. There are positive constants $\varepsilon_{3}$ and $T$ such that for all $r_{0} \in B_{0}\left(\varepsilon_{3}, H^{s_{1}+1}\left(\Gamma_{0}\right)\right)$ the problem (6.2) has at most one solution in

$$
C^{1}\left(I T, H^{s_{1}+1}\left(\Gamma_{0}\right)\right) \cap L^{\infty}\left(I T, H^{s_{1}+\frac{3}{2}}\left(\Gamma_{0}\right)\right) .
$$

Proof. Let $\varepsilon_{3}$ be small enough that, due to Lemmas 9 and 10, (6.3) and (6.7) hold for $s=s_{1}$ if $\|r\|_{s_{1}+1}^{\Omega_{0}} \leq 2 \varepsilon_{3}$. Suppose $r, v \in C^{1}\left(I T, H^{s_{1}+1}\left(\Gamma_{0}\right)\right) \cap L^{\infty}\left(I T, H^{s_{1}+\frac{3}{2}}\left(\Gamma_{0}\right)\right)$ are solutions of (6.2). From (6.3) one concludes $\|r(t)\|_{s_{1}+1}^{\Omega_{0}},\|v(t)\|_{s_{1}+1}^{\Omega_{0}} \leq 2 \varepsilon_{3}$ for all $t \in I T$ for a certain $T>0$ in the same manner as the corresponding estimates on the $r_{j}$ in the proof of Proposition 1. Moreover, using the boundedness of $\|r(t)\|_{s+\frac{3}{2}}^{\Gamma_{0}},\|v(t)\|_{s+\frac{3}{2}}^{\Gamma_{0}}$ and the generalized Schwarz inequality we find from (6.7)

$$
\begin{aligned}
\frac{d}{d t}\left(\|r(t)-v(t)\|_{s_{1}+1}^{\Gamma_{0}{ }^{2}}{ }^{2}\right)= & 2(\rho(r(t))-\rho(v(t)), r(t)-v(t))_{s_{1}+1} \\
\leq & -c\|r(t)-v(t)\|_{s_{1}+\frac{3}{2}}^{\Gamma_{0}}{ }^{2}+C\|r(t)-v(t)\|_{s_{1}+\frac{1}{2}}^{\Gamma_{0}}{ }^{2} \\
& +C_{r, v}\|r(t)-v(t)\|_{s_{1}+1}^{\Gamma_{0}}\|r(t)-v(t)\|_{s_{1}+\frac{3}{2}}^{\Gamma_{0}} \\
\leq & C_{r, v}\|r(t)-v(t)\|_{s_{1}+1}^{\Gamma_{0}{ }^{2}}
\end{aligned}
$$

for almost all $t \in I T$ and from the Gronwall inequality it follows $r(t)=v(t)$ for all $t \in I T$ 
In a similar way, under slightly stronger smoothness assumptions on the initial condition, one can prove continuous dependence of $r(t)$ for fixed $t$ on $r(0)$.

\section{Global existence and stability of solutions near the ball}

From physical reasons and corresponding results in the two-dimensional case (partly in the case of the corresponding problem for an outer domain $[3,4,18])$ one expects that the only stationary solutions of our free boundary problem are given by the balls. This will be proved in the following. We remind that $u_{0}$ is the first component of the solution of (2.6) with $\Omega=\Omega_{0}$.

Lemma 11 (Stationary solutions). If $u_{0} \cdot n \equiv 0$ on $\Gamma_{0}$, then $\Gamma_{0}$ has constant mean curvature, i.e. $\Omega_{0}$ is a circle if $N=2$ and a ball if $N=3$.

Proof. From

$$
\int_{\Gamma_{0}} \kappa_{0} n \cdot u_{0} d \Gamma_{0}=\int_{\Gamma_{0}} \mathcal{T}\left(u_{0}, p_{0}\right) n \cdot u_{0} d \Gamma_{0}=0
$$

it follows by setting $u=v=u_{0}, p=p_{0}$ in (2.3) that, using the notation of Section 2, $a\left(u_{0}, u_{0}\right)=0$ and thus $u_{0}=0$. The Stokes equations and the boundary condition on the stress tensor reduce to

$$
\left.\begin{array}{ccc}
\nabla p=0 & \text { in } & \Omega_{0} \\
-p n=\kappa_{0} n & \text { on } & \Gamma_{0}
\end{array}\right\},
$$

hence both $p$ and $\kappa_{0}$ are constant. This completes the proof because the only (bounded) simply connected domains in $\mathbb{R}^{3}$ whose boundaries have constant mean curvature are the balls (see, e.g., [5])

In order to investigate the moving boundary problem near the ball we set

$$
\Omega_{0}=B_{0}\left(1, \mathbb{R}^{N}\right) \quad \text { and } \quad \zeta(\xi)=n(\xi) .
$$

This clearly satisfies all assumptions made above and leads to $\gamma \equiv 1, \kappa_{0}=-(N-1)$ and $\Gamma_{0}=S^{N-1}$. The diffeomorphism $\Phi_{0}$ can and will be chosen to be the identity and thus we have $R_{0}=\mathcal{R}_{0} \equiv 1$ on $\Gamma_{0}$. In this case we get from (4.6)

$$
D^{\alpha} \rho_{1}(r)=\rho_{1}\left(D^{\alpha} r\right)
$$

For the sake of technical simplicity, the following considerations are restricted to the case $N=3$. They can be generalized, however, to the general case without essential changes.

Using spherical coordinates it is not difficult to obtain the expressions

$$
\begin{aligned}
V(r) & =\frac{1}{3} \int_{\Gamma_{0}}(1+r)^{3} d \Gamma_{0} \\
M(r) & =\frac{1}{4} \int_{\Gamma_{0}}(1+r)^{4} n d \Gamma_{0}
\end{aligned}
$$


for the volume and the centre of gravity of the domain $\Omega_{r}$, respectively. We define the function $F: H^{s_{0}}\left(\Gamma_{0}\right) \longrightarrow \mathbb{R} \times \mathbb{R}^{3}$ by

$$
F(r)=\left[\begin{array}{c}
V(r)-\frac{4}{3} \pi \\
M(r)
\end{array}\right]
$$

Note that $F$ is an analytic function on $H^{s_{0}}\left(\Gamma_{0}\right), F(0)=0$, and

$$
F^{\prime}(0)[h]=\left[\begin{array}{c}
\int_{\Gamma_{0}} h d \Gamma_{0} \\
\int_{\Gamma_{0}} h n d \Gamma_{0}
\end{array}\right] .
$$

For all $s \geq s_{0}$ we define

$$
\mathcal{M}_{s}=\left\{r \in H^{s}\left(\Gamma_{0}\right) \mid F(r)=0\right\}
$$

and demand $r_{0} \in \mathcal{M}_{s+1}$ in (6.2). (It is obvious that this, as well as the choice of radius 1 , is no essential restriction of generality but just a matter of appropriate shifting and scaling.) Because of the incompressibility condition and the demand $\int_{\Omega} u d x=0$ in the fixed time problem we find simply by integration

$$
r(t) \in \mathcal{M}_{s+1} \quad \forall t \in I T
$$

for any solution of (6.2).

For the linearization we find after a certain amount of calculation $\rho_{1}(r)=\left(\operatorname{Tr}_{\Gamma_{0}} \dot{u}^{\star}\right) \cdot n$ where $\left(\dot{u}^{\star}, \dot{p}^{\star}, \dot{\lambda}^{\star}\right)$ is the solution of the variational problem

$$
\begin{aligned}
& a\left(\dot{u}^{\star}, v\right)+b\left(v, \dot{p}^{\star}\right)+\dot{\lambda}_{1}^{\star T} \varphi_{1}(v)+\dot{\lambda}_{2}^{\star T} \varphi_{2}(v)= \int_{\Gamma_{0}}\left(\Delta_{\Gamma_{0}} r+2 r\right) n \cdot v d \Gamma_{0} \\
& \text { for all } v \in\left(H^{1}(\Omega)\right)^{N} \\
& b\left(\dot{u}^{\star}, q\right)=0 \quad \forall q \in L^{2}(\Omega) \\
& \varphi_{1}\left(\dot{u}^{\star}\right)=0 \\
& \varphi_{2}\left(\dot{u}^{\star}\right)=0 .
\end{aligned}
$$

Note that

$$
\dot{\lambda}_{1}^{\star}=\dot{\lambda}_{2}^{\star}=0
$$

and thus we get, instead of (5.4), the sharper estimate

$$
\left\|\dot{p}^{\star}\right\|_{0}^{\Omega_{0}} \leq C\left\|\dot{u}^{*}\right\|_{1}^{\Omega_{0}} .
$$

In the following we use series expansions in eigenfunctions of the Laplace-Beltrami operator on $S^{2}$ to define Hilbert norms that are adjusted to our needs. Let $\left\{Y_{k l} \mid l=\right.$ $0,1,2, \ldots ; k=-l, \ldots, l\}$ be an orthonormal basis of $L^{2}\left(S^{2}\right)$ satisfying $\Delta_{\Gamma_{0}} Y_{k l}=-l(l+$ 1) $Y_{k l}$. Such a basis is given by choosing an arbitrary $L^{2}$-orthonormal basis of the $l$-th 
order spherical harmonics for all $l$. We will write $r_{k l}=\left(r, Y_{k l}\right)_{0}$ and introduce (on all $\left.H^{s}\left(\Gamma_{0}\right)\right)$ the projection $\mathcal{P}$ by

$$
\mathcal{P} r=\sum_{l=2}^{\infty} \sum_{k=-l}^{l} r_{k l} Y_{k l}
$$

and on the spaces $H^{s}\left(\Gamma_{0}\right)$ for positive integer $s, s=-\frac{1}{2}$, and $s=\frac{3}{2}$ the scalar products

$$
\begin{aligned}
& (r, v)_{s}=r_{00} v_{00}+\sum_{k=-1}^{1} r_{k 1} v_{k 1}+\sum_{l=2}^{\infty} \sum_{k=-l}^{l}(l(l+1)-2)^{s} r_{k l} v_{k l} \quad(s<2) \\
& (r, v)_{s}=r_{00} v_{00}+\sum_{k=-1}^{1} r_{k 1} v_{k 1}+\sum_{|\alpha| \leq s-1}\left(D_{--}^{\alpha} \mathcal{P} r, D^{\alpha} \mathcal{P} v\right)_{1} \quad(s \geq 2) .
\end{aligned}
$$

It is easily seen that $\mathcal{P}$ commutes with all $D^{\alpha}$ and that $\mathcal{P}$ is orthogonal with respect to all these scalar products. Furthermore, we introduce a semi-scalar product and a seminorm on $H^{s}\left(\Gamma_{0}\right)$ by

$$
\begin{aligned}
{[r, v]_{s} } & =(\mathcal{P} r, \mathcal{P} v)_{s} \\
|r|_{s} & =[r, r]_{s}^{\frac{1}{2}} .
\end{aligned}
$$

Lemma 12. Assume $s \geq s_{1}$. There are constants $\varepsilon>0$ and $C>0$ (depending on s) such that

$$
\|r\|_{s}^{\Gamma_{0}} \leq C\left(|r|_{s}+\|F(r)\|_{\mathbb{R} \times \mathbb{R}^{3}}\right)
$$

for all $r \in B_{0}\left(\varepsilon, H^{s}\left(\Gamma_{0}\right)\right)$ and, moreover,

$$
\|r\|_{s}^{\Gamma_{0}} \leq\left(1+C|r|_{s}\right)|r|_{s}
$$

for all $r \in \mathcal{M}_{s} \cap B_{0}\left(\varepsilon, H^{s}\left(\Gamma_{0}\right)\right)$.

Proof. The first inequality is a consequence of the local diffeomorphism theorem applied to the mapping $\Phi: H^{s}\left(\Gamma_{0}\right) \longrightarrow \mathcal{P}\left[H^{s}\left(\Gamma_{0}\right)\right] \times\left(\mathbb{R} \times \mathbb{R}^{3}\right)$ defined by $\Phi(r)=\left[\begin{array}{c}\mathcal{P}_{r} \\ F(r)\end{array}\right]$ in the neighbourhood of 0 .

Due to the orthogonality of $\mathcal{P}$ we have $\|r\|_{s}^{\Gamma_{0}^{2}}=|r|_{s}^{2}+\|\bar{r}\|_{s}^{\Gamma_{0}^{2}}$ with $\bar{r}=(I-\mathcal{P}) r$. We consider now $\bar{r} \in \operatorname{span}\left\{1, x_{1}, x_{2}, x_{3}\right\}$ as solution of the equation

$$
\tilde{F}(\mathcal{P} r, \bar{r})=F(\mathcal{P}(r)+\bar{r})=0
$$

which is satisfied for all $r \in \mathcal{M}_{s}$. Applying the implicit function theorem to it and using that the Fréchet derivative of $\widetilde{F}$ with respect to the first argument at $(0,0)$ is the zero operator we find $\|\bar{r}\|_{s}^{\Gamma_{0}} \leq C|r|_{s}^{2}$ if $|r|_{s}$ is sufficiently small. The estimate (7.7) follows easily from this

The following estimates are parallel to those given in the Lemmas 8 and 9. The key idea here is that, due to the new context and the use of the seminorms $|\cdot|_{\mathrm{s}}$ instead of complete norms, one is able to avoid the occurrence of "lower order terms". 
Lemma 13. Under the additional assumptions (7.1), there is a constant $c>0$ such that

$$
-\left[\rho_{1} r, r\right]_{1} \geq c|r|_{\frac{3}{2}}^{2}
$$

holds for all $r \in H^{2}\left(\Gamma_{0}\right)$.

Proof. Taking into account that

$$
\left(\rho_{1} r\right)_{00}=(4 \pi)^{-\frac{1}{2}} \int_{\Gamma_{0}} \dot{u}^{\star} \cdot n d \Gamma_{0}=(4 \pi)^{-\frac{1}{2}} \int_{\Omega_{0}} \operatorname{div} \dot{u}^{\star} d x=0
$$

we find

$$
\begin{aligned}
-\left[\rho_{1} r, r\right]_{1} & =-\sum_{l=2}^{\infty} \sum_{k=-l}^{l}(l(l+1)-2)\left(\rho_{1} r\right)_{k l} r_{k l} \\
& =-\sum_{l=0}^{\infty} \sum_{k=-l}^{l}(l(l+1)-2)\left(\rho_{1} r\right)_{k l} r_{k l} \\
& =-\sum_{l=0}^{\infty} \sum_{k=-l}^{l}\left(\rho_{1} r\right)_{k l}\left(-\Delta_{\Gamma_{0}} r-2 r\right)_{k l} \\
& =\int_{\Gamma_{0}} \rho_{1} r\left(\Delta_{\Gamma_{0}} r+2 r\right) d \Gamma_{0} \\
& =\int_{\Gamma_{0}} \dot{u}^{\star}\left(\Delta_{\Gamma_{0}} r+2 r\right) d \Gamma_{0} \\
& =a\left(\dot{u}^{\star}, \dot{u}^{\star}\right) \\
& \geq c\left\|\dot{u}^{\star}\right\|_{1}^{\Omega_{0}{ }^{2}} .
\end{aligned}
$$

On the other hand,

$$
|r|_{\frac{3}{2}}^{2}=\left|\Delta_{\Gamma_{0}} r+2 r\right|_{-\frac{1}{2}}^{2} \leq\left\|\Delta_{\Gamma_{0}} r+2 r\right\|_{-\frac{1}{2}}^{\Gamma_{0}{ }^{2}} \leq C\left\|\left(\dot{u}^{\star}, \dot{p}^{\star}, \dot{\lambda}^{\star}\right)\right\|_{X}^{2}
$$

where the last inequality can be shown analogously to the general case (cf. (5.7), $\Delta_{\Gamma_{0}} r$ has to be replaced by $\Delta_{\Gamma_{0}} r+2 r$ ). Taking into account now (7.4) and (7.5) completes the proof

Lemma 14. Under the additional assumptions (7.1), for all integer $s \geq s_{1}$ the inequality

$$
[\rho(r), r]_{s+1} \leq-c|r|_{s+1}^{2}+C\|F(r)\|_{\mathbb{R} \times \mathbb{R}^{3}}^{2}
$$

holds for all $r \in B_{0}\left(\varepsilon, H^{s+1}\left(\Gamma_{0}\right)\right) \cap H^{s+2}\left(\Gamma_{0}\right)$ where the positive constants $\varepsilon, c$, and $C$ depend only on $s$.

Proof. In analogy to the proof of Lemma 9, we have

$$
[\rho(r), r]_{s+1}=\sum_{|\alpha| \leq s}\left[D^{\alpha} \rho(r), D^{\alpha} r\right]_{1}
$$


and estimate the summands on the right as in Lemma 10 where we take additionally into account $(7.2)$ and $\rho_{0}=\rho(0)=0$. Thus we get

$$
\begin{aligned}
{\left[D^{\alpha} \rho(r), D^{\alpha} r\right]_{1} } & =\left[D^{\alpha} \rho_{1} r, D^{\alpha} r\right]_{1}+\sum_{k=2}^{\infty}\left[D^{\alpha} \rho_{k}(r, \ldots, r), D^{\alpha} r\right]_{1} \\
& \leq-c\left|D^{\alpha} r\right|_{\frac{3}{2}}^{2}+\sum_{k=2}^{\infty} C_{k}\|r\|_{s+1}^{\Gamma_{0}+1}\|r\|_{s+\frac{3}{2}}^{\Gamma_{0}}{ }^{k-1}
\end{aligned}
$$

Summing up and using Lemma 12 we find

$$
\begin{aligned}
{[\rho(r), r]_{s+1} } & \leq-c \sum_{|\alpha| \leq s}\left|D^{\alpha} r\right|_{\frac{3}{2}}^{2}+C \sum_{k=2}^{\infty} C_{k}\|r\|_{s+1}^{\Gamma_{0}{ }^{k-1}}\|r\|_{s+\frac{3}{2}}^{\Gamma_{0}}{ }^{2} \\
& \leq-c \sum_{|\alpha| \leq s}\left|D^{\alpha} r\right|_{\frac{3}{2}}^{2}+C \sum_{k=2}^{\infty} C_{k}\|r\|_{s+1}^{\Gamma_{0}{ }^{k-1}}\left(|r|_{s+\frac{3}{2}}+\|F(r)\|_{\mathbb{R} \times \mathbb{R}^{3}}\right)^{2} \\
& \leq-c \sum_{|\alpha| \leq s}\left\|D^{\alpha} \operatorname{Pr}\right\|_{\frac{3}{2}}^{\Gamma_{0}{ }^{2}}+C \sum_{k=2}^{\infty} C_{k}\|r\|_{s+1}^{\Gamma_{0}{ }^{k-1}}\left(|r|_{s+\frac{3}{2}}^{2}+\|F(r)\|_{\mathbb{R} \times \mathbb{R}^{3}}^{2}\right)
\end{aligned}
$$

and the proof is completed by using $\sum_{|\alpha| \leq s}\left\|D^{\alpha} \operatorname{Pr}_{\underline{3}}^{\Gamma_{0}{ }^{2}} \geq c\right\| \mathcal{P r} \|_{s+\frac{3}{2}}^{\Gamma_{0}}{ }^{2}$ and making a suitable choice for $\varepsilon$

Proposition 3 (Global existence and exponential stability near equilibrium). $U n$ der the assumptions of Proposition 1 and (7.1), $s \geq s_{1}, r_{0} \in \mathcal{M}_{s+1} \cap B_{0}\left(\varepsilon, H^{s+1}\left(\Gamma_{0}\right)\right)$ with $\varepsilon$ sufficiently small (depending on $s$ ), the initial value problem (6.2) has a solution

$$
r \in C_{w}\left(\mathbb{R}_{+}, H^{s+1}\left(\Gamma_{0}\right)\right) \cap C_{w}^{1}\left(\mathbb{R}_{+}, H^{s}\left(\Gamma_{0}\right)\right)
$$

that satisfies the estimate

$$
\|r(t)\|_{s+1}^{\Gamma_{0}} \leq C e^{-c t}\left\|r_{0}\right\|_{s+1}^{\Gamma_{0}} \quad \forall t \geq 0
$$

with a positive constant $c$ depending only on $s$.

Proof. Note that (7.8) implies (6.3) for all $r \in H^{s+2}\left(\Gamma_{0}\right)$ with $\|r\|_{9+1}^{\Gamma_{0}}$ sufficiently small. We choose $\tilde{\varepsilon}$ small enough that both (6.3) and (7.7) with $s$ replaced by $s+1$ holds if $\|r\|_{s+1}^{\Gamma_{0}} \leq 2 \tilde{\varepsilon}$. We assume $\left\|r_{0}\right\|_{s+1}^{\Gamma_{0}} \leq \tilde{\varepsilon}$ and proceed as in the proof of Proposition 1 , working only with estimates in $H^{s+1}\left(\Gamma_{0}\right)$. We choose the finite-dimensional subspaces $M_{j} \subset H^{s+2}\left(\Gamma_{0}\right)$ such that $M_{1}=\operatorname{span}\left\{1, x_{1}, x_{2}, x_{3}\right\}$ and $\overline{\operatorname{span} \bigcup_{j>1} M_{j}}=\mathcal{P}\left[H^{s+2}\left(\Gamma_{0}\right)\right]$.

Thus, there is a $T>0$ such that (6.2) has a solution $r$ in $C_{w}\left(I T, H^{s+1}\left(\Gamma_{0}\right)\right) \cap$ $C_{w}^{1}\left(I T, H^{s}\left(\Gamma_{0}\right)\right)$. We set $\varepsilon=\min \left(\tilde{\varepsilon}, \frac{e^{\frac{c T}{2}}-1}{C^{*}}\right)$ where $c$ and $C^{*}$ are the constants from (7.8) and (7.7), respectively.

The solution $r$ is given by

$$
r(t)=w-\lim _{j \rightarrow \infty} r_{j}(t) \quad \forall t \in I T
$$


where $w$-lim denotes the the weak limit in $H^{s+1}\left(\Gamma_{0}\right)$, the $r_{j} \in C^{1}\left(I T, H^{s+2}\left(\Gamma_{0}\right)\right)$ are the solutions of the Galerkin equations (6.4), and the convergence is uniform in $t$. Hence $r_{j}(t) \stackrel{H^{\circ}\left(\Gamma_{0}\right)}{\longrightarrow} r(t)$ uniformly in $t$ and thus

$$
\left\|F\left(r_{j}(t)\right)\right\|_{\mathbb{R} \times \mathbb{R}^{3}} \rightarrow 0 \quad \text { uniformly in } t \in I T
$$

because, as remarked above, $r(t) \in \mathcal{M}_{s+1}$.

Our choice of the $M_{j}$ yields that $\mathcal{P}$ and $P_{j}$ commute for all $j$, and thus we have for all $t \in I T$

$$
\begin{aligned}
\frac{1}{2} \frac{d}{d t}\left(\left|r_{j}(t)\right|_{s+1}^{2}\right) & =\left[P_{j} \rho\left(r_{j}(t)\right), r_{j}(t)\right]_{s+1} \\
& =\left(\mathcal{P} P_{j} \rho\left(r_{j}(t)\right), \mathcal{P} r_{j}(t)\right)_{s+1} \\
& =\left(\mathcal{P} \rho\left(r_{j}(t)\right), \mathcal{P} P_{j} r_{j}(t)\right)_{s+1} \\
& =\left[\rho\left(r_{j}(t)\right), r_{j}(t)\right]_{s+1} \\
& \leq-c\left|r_{j}(t)\right|_{s+1}^{2}+C\left\|F\left(r_{j}(t)\right)\right\|_{\mathbb{R} \times \mathbb{R}^{3}}^{2}
\end{aligned}
$$

because of $P_{j} r_{j}=r_{j},\left\|r_{j}(t)\right\|_{s+1}^{\Gamma_{0}} \leq 2 \tilde{\varepsilon}$, and (7.8), hence

$$
\left|r_{j}(t)\right|_{s+1}^{2} \leq e^{-c t}\left|r_{0}\right|_{s+1}^{2}+C \int_{0}^{t} e^{c(\tau-t)}\left\|F\left(r_{j}(\tau)\right)\right\|_{\mathbb{R} \times \mathbb{R}^{3}}^{2} d \tau
$$

and thus, using (7.10),

$$
\begin{aligned}
|r(t)|_{s+1} & =\|\mathcal{P r}(t)\|_{s+1}^{\Gamma_{0}}=\left\|\mathcal{P} w-\lim _{j \rightarrow \infty} r_{j}(t)\right\|_{s+1}^{\Gamma_{0}}=\left\|w-\lim _{j \rightarrow \infty} \mathcal{P} r_{j}(t)\right\|_{s+1}^{\Gamma_{0}} \\
& \leq \liminf _{j \rightarrow \infty}\left\|\mathcal{P} r_{j}(t)\right\|_{s+1}^{\Gamma_{0}}=\liminf _{j \rightarrow \infty}\left|r_{j}(t)\right|_{s+1} \leq e^{-c t}\left|r_{0}\right|_{s+1} .
\end{aligned}
$$

Finally, $r(t) \in \mathcal{M}_{s+1} \cap B_{0}\left(2 \varepsilon, H^{s+1}\left(\Gamma_{0}\right)\right)$ implies

$$
\begin{aligned}
\|r(T)\|_{s+1}^{\Gamma_{0}} & \leq\left(1+C^{*}|r(T)|_{s+1}\right)|r(T)|_{s+1} \\
& \leq\left(1+C^{*}\left\|r_{0}\right\|_{s+1}^{\Gamma_{0}}\right) e^{-c T}\left\|r_{0}\right\|_{s+1}^{\Gamma_{0}} \\
& \leq e^{-\frac{c}{2} T}\left\|r_{0}\right\|_{s+1}^{\Gamma_{0}} \\
& \leq \varepsilon
\end{aligned}
$$

because of $\varepsilon \leq \frac{e^{\frac{c T}{2}}-1}{C^{*}}$. Therefore we can continue the solution to $[T, 2 T]$ and by induction to $[n T,(n+1) T]$ for all $n \in \mathbb{N}$ with the estimate

$$
|r(t)|_{s+1} \leq e^{-c t}\left|r_{0}\right|_{s+1} \quad \forall t \geq 0
$$

from which (7.9) follows by (7.6) 


\section{Conclusions}

The most remarkable feature of the analysis given above is that it does not depend too strongly on special properties of the Stokes operator: The only facts we have used about it are its rotational invariance and ellipticity in the sense of Agmon-Douglis-Nirenberg, together with the regularity and self-adjointness of the corresponding Neumann problem. Therefore it seems to be possible to apply the same methods without essential changes to similar non-local evolution problems, in particular, to the problem of Hele-Shaw flow driven by surface tension.

Based on discussion of perturbations of the liquid domain and linearization of the resulting operator with respect to these perturbations, one can also obtain existence, uniqueness and smoothness results for stationary free boundary problems for the full Navier-Stokes equations $[1,2]$.

It has to be pointed out that the assumptions of the general existence theorem from [15] that has been applied here does not resemble the parabolic character of the evolution equation, actually, it is more suited to nonlinear hyperbolic equations (and has originally been used for a problem of that kind). This is the reason that our approach provides no proof of the smoothing effect we expect to find in a parabolic problem.

Finally, we remark that due to the local character the analysis given here obviously cannot provide answers to the questions on the occurrence of irregular behaviour like cusp formation or change of connectivity.

Acknowledgements. The second author is indebted to Prof. J. de Graaf for a lot of stimulating discussions.

\section{References}

[1] Abergel, F.: A geometric approach to the study of stationary free surface flows for viscous liquids. Proc. Royal Soc. Edinburgh 123A (1993), $209-229$.

[2] Abergel, F. and E. Rouy: Interfaces stationnaires pour les équations de Navier-Stokes. Inst. Nat. Rech. Inf. Aut. (INRIA) Research Report No. 2651 (1995), pp. 45.

[3] Antanovskii, L. K.: Analyticity of a free boundary in plane quasi-steady flow of a liquid form subject to variable surface tension. Lect. Notes Math. 1530 (1993), 1 - 16.

[4] Antanovskii, L. K.: Creeping thermocapillary motion of a two-dimensional deformable bubble: existence theorem and numerical simulation. Eur. J. Mech./Issue B: Fluids 11 (1992), $741-758$.

[5] Blaschke, W. and K. Leichtweiß: Elementare Differentialgeometrie. Berlin et al.: SpringerVerlag 1973.

[6] Brezzi, F. and M. Fortin: Mixed and Hybrid Finite Element methods. Berlin et al.: Springer-Verlag 1991.

[7] Chen, X.: The Hele-Shaw problem and area-preserving curve-shortening motions. Arch. Rat. Mech. Anal. 123 (1993), 117 - 151.

[8] Constantin, P. and L. Kadanoff: Dynamics of a complex interface. Physica 47D (1991), $450-460$. 
[9] Constantin, P. and M. Pugh: Global solution for small data to the Hele-Shaw problem. Nonlinearity 6 (1993), $393-415$.

[10] Courant, R. and D. Hilbert: Methoden der mathematischen Physik. Vol. 2. Berlin et al.: Springer-Verlag 1968.

(11) Fichera, G.: Existence theorems in elasticity. In: Handbuch der Physik, Vol. VIa/II (ed.: S. Flügge). Berlin et al.: Springer-Verlag 1972.

[12] Galdi, G. P.: An Introduction to the Mathematical Theory of Navier-Stokes-Equations, Vol. I (Springer Tracts in Natural Philosophy: Vol. 38). New York et. al.: SpringerVerlag 1994.

[13] Günther, M. and G. Prokert: Existence results for the quasistationary motion of a viscous capillary liquid drop. Preprint. Eindhoven: Univ. of Technology, Preprint RANA 96-01 (1996), pp. 55.

[14] Hopper, R. W.: Plane Stokes flow driven by capillarity on a free surface. J. Fluid Mech. $213(1990), 349-375$.

[15] Kato, T. and C. Y. Lai: Nonlinear evolution equations and the Euler flow. J. Funct. Anal. 56 (1984), $15-28$.

[16] Klein Obbink: Moving Boundary Problems in Relation with Equations of Löuner-Kufarev Type. PhD Thesis. Eindhoven: University of Technology 1995.

[17] Ladyshenskaja, O. A.: Funktionalanalytische Untersuchungen der Navier-Stokes-Gleichungen. Berlin: Dt. Verlag Wiss. 1965.

[18] Prokert, G.: On the existence of solutions in plane quasistationary Stokes flow driven by surface tension. Euro. J. Appl. Math. 6 (1995), $539-558$.

[19] Seeley, R.: Topics in pseudo-differential operators. In: Pseudo-differential Operators (ed.: L. Nirenberg). Centro Intern. Mat. Estivo (C.I.M.E.) Proceedings, Rome 1969, pp. 169 -305 .

[20] Triebel, H.: Höhere Analysis. Berlin: Dt. Verlag Wiss. 1972.

[21] Vorst, G. A. L. v. d.: Modelling and Numerical Simulation of Viscous Sintering. PhD Thesis. Eindhoven: University of Technology 1994.

[22] Zeidler, E.: Vorlesungen über nichtlineare Funktionalanalysis. Vol. I: Fixpunktsätze. Leipzig: B. G. Teubner Verlagsges. 1976. 